\title{
Centaur and giant planet crossing populations: origin and distribution
}

\author{
Romina P. Di Sisto · Natalia L. Rossignoli
}

\begin{abstract}
The current giant planet region is a transitional zone where transneptunian objects (TNOs) cross in their way to becoming Jupiter Family Comets (JFCs). Their dynamical behavior is conditioned by the intrinsic dynamical features of TNOs and also by the encounters with the giant planets. We address the Giant Planet Crossing (GPC) population (those objects with $5.2 \mathrm{au}$ $<q<30 \mathrm{au}$ ) studying their number and their evolution from their sources, considering the current configuration of the Solar System. This subject is reviewed from previous investigations and also addressed by new numerical simulations of the dynamical evolution of Scattered Disk Objects (SDOs). We obtain a model of the intrinsic orbital element distribution of GPCs. The Scattered Disk represents the main source of prograde GPCs and Centaurs, while the contribution from Plutinos lies between one and two orders of magnitude below that from the SD. We obtain the number and size distribution of GPCs from our model, computing $9600 \mathrm{GPCs}$ from the SD with $D>100 \mathrm{~km}$ and $\sim 10^{8}$ with $D>1 \mathrm{~km}$ in the current population. The contribution from other sources is considered negligible. The mean lifetime in the Centaur zone is 7.2 Myr, while the mean lifetime of SDOs in the GPC zone is of $68 \mathrm{Myr}$. The latter is dependent on the initial inclination, being the ones with high inclinations the ones that survive the longest in the GPC zone. There is also a correlation of lifetime with perihelion distance, where greater perihelion leads to longer lifetime. The dynamical evolution of observed GPCs is different for prograde and retrograde objects. Retrograde GPCs have lower median lifetime than prograde ones, thus experiencing a comparatively faster evolution. However, it is probable that this faster evolution is due to the fact that the majority of retrograde GPCs have low perihelion values and then, lower lifetimes.
\end{abstract}

Keywords Centaurs · Numerical methods · Transneptunian Objects

Romina P. Di Sisto

Facultad de Ciencias Astronómicas y Geofísicas, Universidad Nacional de La Plata Instituto de Astrofísica de La Plata, CCT La Plata-CONICET-UNLP

Paseo del Bosque S/N (1900), La Plata, Argentina.

E-mail: romina@fcaglp.unlp.edu.ar

Natalia L. Rossignoli

Instituto de Astrofísica de La Plata, CCT La Plata-CONICET-UNLP

Paseo del Bosque S/N (1900), La Plata, Argentina. 


\section{Introduction}

The giant planet region is a vast territory in the Solar System in which it is possible and suitable to test both the Solar System origin and its evolution, as well as specific dynamical and physical processes that take place in the planets, their satellites and in all the minor body populations. In addition, the whole subject is continually fed by new observations and theoretical studies.

The dynamical evolution of objects in the giant planet region, commonly called "Centaurs" has been mainly investigated in relation to their "parental ties", i.e. as progeny of Transneptunian Objects (TNOs) and as progenitors of Jupiter Family Comets (JFCs). Due to their transitional object quality, their dynamical behavior is conditioned by the intrinsic dynamical features of TNOs. Neptune can be considered the "nexus" between TNOs and Centaurs, since the eventual gravitational interactions of some TNOs with this planet can transfer them to the planetary zone, becoming then Centaur objects. Although the evolutionary path of TNOs from the transneptunian (TN) region into the Centaur region and then into the JFC population is well studied and accepted, the actual number and size distribution of Centaurs remains unclear.

The boundary between the TN and Centaur regions and the dynamical definition of Centaurs are somewhat variable in the literature, and this hinders the comparison between different studies. Additionally, there are few observational estimates of the Centaur population from a well-characterized survey. On the contrary, TNOs and JFCs are much more observed and their size distribution and number are more constrained. Therefore, the predictions on Centaurs are mostly based on a TNO-JFC steady-state. The existence of a TN region as a source of JFCs was first suggested by Edgeworth (1938) and Kuiper (1951); but it was not until the paper by Fernández (1980) that this matter was theoretically analyzed. In this work, Fernández proposed a transneptunian belt between $\sim 35$ and 50 au as an alternative, more efficient JFC source (compared to the Oort Cloud). Later, Duncan et al (1988) addressed this problem through numerical simulations. The next two baseline papers that analyzed the TNOs as a source of JFCs were the studies by Levison and Duncan (1997) and Duncan and Levison (1997). In the first paper the authors studied the evolution of 20 now called Classical Transneptunian Objects (CTNOs) plus clones, and found that some objects in their simulation crossed the orbit of Neptune and were scattered by this planet. Thus, they inferred the existence of an excited population in the $\mathrm{TN}$ region that could be an order of magnitude larger than the Classical Belt, which they called the Scattered Disk (SD). In the second paper, the authors suggested that the SD should produce more Ecliptic Comets (ECs) (a group that includes JFCs as well as Centaurs) than the Classical Belt because SDOs can approach Neptune during their perihelion passages and be scattered by this planet to orbits with shorter orbital periods.

As the structure of the TN region emerged thanks to the observations, four different sub-populations were identified. The classical transneptunian objects (CTNOs) with semimajor axes between 40 au $\lesssim a \lesssim 50$ au and orbits with both low eccentricities and low inclinations, the resonant objects in mean- 
motion resonances (MMRs) with Neptune, such as the Plutinos in 2:3 MMR, the scattered disk objects (SDOs) with perihelion distances between $30 \mathrm{au}$ $<q \lesssim 39$ au that can cross the orbit of Neptune and eventually evolve into the planetary region becoming a Centaur, and the detached objects with $q>39$ au that are decoupled from Neptune.

It became evident that the structure and dynamical characteristics of the TN sub-populations showed hints of a convulsed past. New planetary formation models based on planetary migration (e.g. Fernandez and Ip (1984); Malhotra (1993, 1995); Tsiganis et al (2005); Walsh et al (2011);

Nesvorný and Morbidelli (2012); Nesvorný (2015)) were necessary to explain the observations. Many studies have addressed the matter since the pioneering work of Fernandez and Ip (1984), where the authors found a radial displacement for Uranus and Neptune during their accretion and scattering of planetesimals, and the mechanism of radial migration came to light. Malhotra (1993, 1995) succeeded in explaining the capture of Pluto into the 3:2 MMR with Neptune, acquiring its high eccentricity and inclination from a migrating Neptune. Then, the Nice model (Tsiganis et al 2005) opened the door to a series of works that focused on explaining the current orbital architecture of the Solar System. The planetary migration led, anyway, to a convulse early Solar System evolution in which a great mass depletion should have occurred. After this, the Solar System began to stabilize into the current form and dynamics, ultimately acquiring the present configuration.

Once the stabilization had taken place, small body populations started interacting and established links between each other. In particular, Centaur objects are in a transient zone, permanently interacting with neighboring regions and populations. Their current population is mostly formed and defined by the contributions of their specific sources, mainly in the current $\mathrm{TN}$ region but also in other small body sources. A valuable review paper on formation, orbital properties, evolution and links between small body populations and reservoirs by Dones et al (2015) would be helpful for an interested reader.

In this paper we are interested in analyzing the current Giant Planet Crossing population as a whole. This comprises objects with perihelion distances $q$ less than that of Neptune. However, Centaurs, in spite of being giant planetary crossers, are often defined more narrowly as objects with semimajor axis $a$ between those of Jupiter and Neptune. Thus, they do not represent the complete population of giant planetary crossers. In this work we define and address:

- Giant Planetary Crossers (GPC): those objects with 5.2 au $<q<30$ au.

- Centaurs: objects with $5.2<a<30$ au.

The limit of $q=5.2 \mathrm{au}$ in the definition of GPC is due to the fact that in the region interior to the orbit of Jupiter, i.e. the Jupiter Family Comets (JFC) zone, the perturbations of the terrestrial planets are necessary for the study of the dynamical evolution of small bodies. Besides, a physical model is also required to account for sublimation (Di Sisto et al 2009). All these factors prevent the study of Jupiter crossers to be handled in the same way than that of the other Giant planets. 
In this paper we address the present day GPC, their dynamical evolution, dynamical lifetimes and their number and source feeding regions. In the next section we address the observed GPC and Centaur population, their observational features and size distribution and perform a dynamical evolution. In Sect. 3 we perform a numerical simulation of the evolution of SDOs and their contribution to GPC and Centaurs and in Sect. 4 we address other secondary sources. In sect. 5] we join together all the contributions to the current GPC and Centaur population, and in the last section we present the conclusions.

\section{The observed GPC and Centaurs}

\subsection{From surveys}

The first Centaur to be discovered was (2060) Chiron by Kowal and Gehrels (1977) on November 4, 1977; the first detection of a moving object between the giant planets. Thirteen years later, Meech and Belton (1990) detected a low-surface-brightness coma for Chiron revealing for the first time the transitional nature of this object. Chiron has an absolute magnitude $H=5.8$ and an estimated diameter of $166 \mathrm{~km}$. It is the largest member of the population. The smallest Centaur discovered up to now is (2015 RK277) with $H=15.5$. (5145) Pholus was the second Centaur to be discovered, in 1992, the same year that the first TNO was discovered (Jewitt et al 1992). Many more were soon detected, but following surveys were focused more on finding TNOs than Centaurs. Therefore, in the present there are no surveys focused on finding only Centaurs. Instead, they generally are serendipitous discoveries from TNO designed surveys. As with all small body populations, we usually model the absolute magnitude $(H)$ distribution of TNOs and Centaurs as following one or more exponential distributions including proposed breaks and or divots in that distribution. Considering the simplest form, as an exponential $N(H) \propto 10^{\alpha H}$, where $\alpha$ is the logarithmic slope which characterizes the population, it is possible to relate it with its size distribution. From the relation between magnitude and diameter: $D=1327.510^{-H / 5} / \sqrt{p_{v}}$, where $p_{v}$ is the albedo, the size distribution would result in a power differential law of the form: $N(D) \propto D^{-q}$, where $q=5 \alpha+1$. The first survey designed to discover and determine the orbits of hundreds of TNOs was the Deep Ecliptic Survey (DES) which worked from 1998 to 2005 observing in VR filter (Elliot et al 2005). This survey followed-up observations of 304 objects which allowed for well-determined orbits and dynamical classifications into the sub-populations of the TN zone, i.e. Classical, Scattered, and mean-motion resonances with Neptune and also Centaurs. Adams et al (2014) accounted for the DES biases and estimated an exponential law magnitude distribution valid for TNOs and Centaurs which has a break at fainter objects. Based on observations of Centaurs which were made in the $7.5<H_{r}<11$ range they inferred a broken law with $\alpha_{1}=1.02 \pm 0.01$ for $H_{r} \lesssim 7.2$ and $\alpha_{2}=0.42 \pm 0.02$ for $H_{r}>7.2$. This gives a number of Centaurs with $H_{r} \lesssim 7$ of $13 \pm 5$. 
A survey that has greatly increased the number of observed TNOs and also Centaurs is the Outer Solar System Origins Survey (OSSOS), which operated between 2013 and 2017 and reported the discovery of 840 objects (Bannister et al 2018). This survey observed in the r-band and in a "w" wideband filter, covering an area of $155 \mathrm{deg}^{2}$ of sky to depths of $m_{r}=24.125 .2$, and was designed to discover TNOs with a careful quantification of the biases. This allowed the OSSOS team to develop a survey simulator to account for OSSOS biases (Lawler et al 2018a). Lawler et al (2018b) used a sample of 68 scattering TNOs (defined by $a>30 \mathrm{au}$ ) and Centaurs $(a<30 \mathrm{au}$ ), discovered mainly by OSSOS, to explore their $H$-distribution by using r-band observations. They assumed that scattering TNOs and Centaurs are part of a dynamically "hot" population with a common origin, since their orbits have been excited to higher inclinations and eccentricities by scattering off Neptune or past/current entanglement with mean-motion resonances. In this sense, their sample of Centaurs plus scattering TNOs has a common size distribution which could be different than those of the other TN populations, since they have a different formation and collisional evolution. This selection of objects allowed also to be sensitive to a much fainter $H_{r}$ (i.e. smaller sizes). In fact, they have $H_{r}$ values of 6 to 14.5 due to the very close pericenter distances of some of the TNOs in the sample. Lawler et al $(2018 \mathrm{~b})$ obtained that both a divot and a knee distribution (see e.g. Fig. 2 in Shankman et al (2016)) fits the data. Their preferred knee distribution has the knee in $H=H_{b}=7.7$ with $\alpha_{1}=0.9$ for $H<H_{b}$ and $\alpha_{2}=0.4$ for $H>H_{b}$, while their preferred divot distribution transitions from bright- $\left(\alpha_{1}=0.9\right)$ to faint-end $\left(\alpha_{2}=0.5\right)$ slopes at $H_{b}=8.3$ with a divot contrast $c=3.2$. By using this last distribution, the authors used the OSSOS Survey Simulator to determine the number of SDOs and Centaurs brighter than a given magnitude $\mathrm{H}$ and estimating an SDO population of $N_{S D O}\left(H_{r}<12\right)=2.7 \pm 0.7 \times 10^{6}$ (which corresponds to $D \gtrsim 20 \mathrm{~km}$ for an albedo of 0.06$)$, and $N_{S D O}\left(H_{r}<8.66\right)=9 \pm 2 \times 10^{4}$ $(D \gtrsim 100 \mathrm{~km})$. Assuming that the size distribution holds for smaller sizes, the authors found that $N_{S D O}\left(H_{r}<18\right)=3 \times 10^{9}(D \gtrsim 1 \mathrm{~km})$. For Centaurs: $N_{C}\left(H_{r}<12\right)=3500_{-1400}^{+1800}$, and $N_{C}\left(H_{r}<8.66\right)=110_{-40}^{+60}$. However, it should be noted that the size distribution at smaller sizes is unknown. Observational analysis of the size distribution of small craters on the satellites of the outer planets have shown that the size distribution of the impactor population could have a new break (see Bierhaus and Dones (2015) for a good summary). This possible break was found in particular in the satellites of Saturn (Kirchoff and Schenk 2010) and on Pluto and Charon (Singer et al 2019) from Cassini and New Horizons observations.

Kirchoff and Schenk (2010) obtained differential slopes from $\sim 2.1$ to 3.67 for small craters on the mid-sized Saturnian satellites. Singer et al (2019) found that crater data from Pluto and Charon indicate a shallow differential slope with $q \sim 1.7$ for craters from $\sim 1$ to $13 \mathrm{~km}$ in diameter corresponding to impactors from $\sim 100 \mathrm{~m}$ to $1 \mathrm{~km}$ in diameter. Small craters observed on the TNO Arrokoth by the New Horizons flyby (Spencer et al 2020) are also consistent with the slopes seen for small craters in the Pluto system. 
Nesvorny et al (2019) used previous models of Solar System evolution with a slow, long-range and grainy migration of Neptune to predict the orbital element distributions of current Centaurs by testing the models using the OSSOS survey simulator. They obtained a good match to the observed OSSOS Centaur orbital distribution. The size distribution of the primordial outer disk was calibrated from Jupiter Trojans and, after running the OSSOS survey simulator, the authors also obtained a good match between their model and the observations. Therefore, they predicted a population of Centaurs of 21,000 \pm 8,000 for $D>10 \mathrm{~km}$ with a size distribution that can be obtained from that of Jupiter Trojans $\left(N(>D) \propto D^{-2.1}\right.$ for $\left.5<D<100 \mathrm{~km}\right)$. From this distribution, the number of Centaurs with $H_{r}<12(D \gtrsim 20 \mathrm{~km})$ would be $\sim 4,900 \pm 1,800$, which is in agreement with the estimation of Lawler et al $(2018 \mathrm{~b})$

The number and size distribution of Centaurs is strongly linked to the number and size distribution of SDOs (i.e. their source in the SD), which can be considered as a sign of their origin.

\subsection{Dynamical evolution of observed GPC}

The observed population of objects in the Giant Planet zone has greatly increased in recent years. In order to study and quantify the evolution of the current observed giant planetary crossing population, we developed a numerical study of its dynamical evolution. There are 432 GPCs $(5.2$ au $<q<30$ $\mathrm{au}$ ) listed in the Minor Planet Center database up to May 1st, 2019. Of these objects, 17 have retrograde orbits and 415 have prograde orbits. The orbital element distribution of all the objects is shown in Fig. 1.

For the simulation we consider these 432 GPCs and calculate the orbital parameters at the common epoch of 2019 April 27th for those few objects whose data differed from this epoch. For each catalogued GPC we build six clones, replacing its original mean anomaly by random values between $0^{\circ}$ and $360^{\circ}$ and perform a numerical integration considering a total of 3024 particles (432 real plus 2592 synthetic) over the age of the Solar System. We use the hybrid integrator EVORB (Fernández et al 2002) with an integration step of 0.2 yrs to follow the dynamical evolution of the particles under the gravitational influence of the Sun (including the masses of the terrestrial planets), the four giant planets and Pluto. Each particle evolves for 4.5 Gyr unless removed from the simulation due to a collision with one of the outer planets, reaching a semimajor axis $a>5000$ au or a position with $r<5.2$ au, i.e. entering the JFC zone where the perturbations of the terrestrial planets are not negligible and a physical model is also required to account for sublimation (Di Sisto et al 2009). In addition, we exclude the plutinos from the simulation.

From the initial 3024 particles, the results of the simulation show that 3 particles collide with a planet (1 with Uranus and 2 with Neptune). 1748 particles $(57.8 \%)$ reach a $>5000$ au where they are considered ejected from the Solar System, 1265 (41.8\%) become JFCs and 8 particles survive the total 

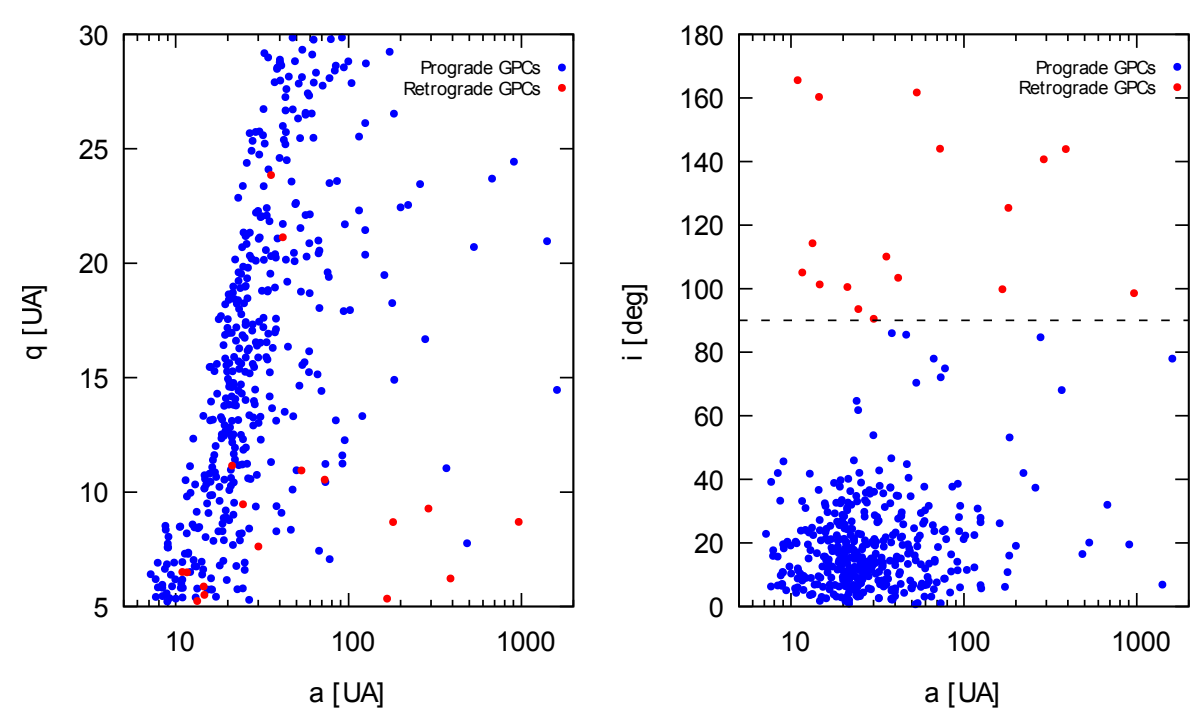

Fig. 1 Semimajor axis vs perihelion (left panel) and semimajor axis vs inclination (right panel) of the catalogued GPCs up to May 1st 2019.

integration time. In addition, we computed the total number of individual encounters between the particles and the outer planets if the particles reached a distance to the planet within their Hill radii. We found that of the total number of encounters with the massive bodies, $0.15 \%$ are with Pluto, $63.51 \%$ are with Neptune, $27.68 \%$ with Uranus, $8.61 \%$ with Saturn and $0.05 \%$ with Jupiter. We further calculated the percentages of the total number of particles that have encounters with the massive bodies during their evolution, obtaining that $22.19 \%$ of the particles have one or more encounters with Pluto, $81.61 \%$ with Neptune, $81.55 \%$ with Uranus, $67.43 \%$ with Saturn and 8.6\% with Jupiter.

Throughout the complete simulation time we recorded the orbital elements of all the described particles every 1,000 years. Our results show that all the integrated GPCs have a median dynamical lifetime of $\sim 16.37 \mathrm{Myr}$ in the GPC zone. In Fig. 2 both the retrograde and prograde GPCs dynamical lifetimes are shown as normalized distributions. The retrograde GPCs have a median dynamical lifetime of $\sim 5.48 \mathrm{Myr}$, which is much shorter than the median dynamical lifetime of $\sim 17.43 \mathrm{Myr}$ of the prograde GPCs. This difference may indicate that the observed retrograde GPCs experience a faster evolution than the observed prograde GPCs. This could be connected with the different perihelion distribution of the observed prograde and retrograde GPCs in our sample. Indeed, it is seen from Fig. 1 that most of the retrograde objects (15 of 17) have perihelion distances of less than $\sim 12$ au. Di Sisto and Brunini (2007) noted that the dynamical evolution in the Giant planetary zone is strongly dependent on the perihelion distance, being the lowest perihelion objects those who evolve faster. Therefore, it is probable that the faster evolution of our initial retrograde GPCs is due to the fact that the majority of them have low 


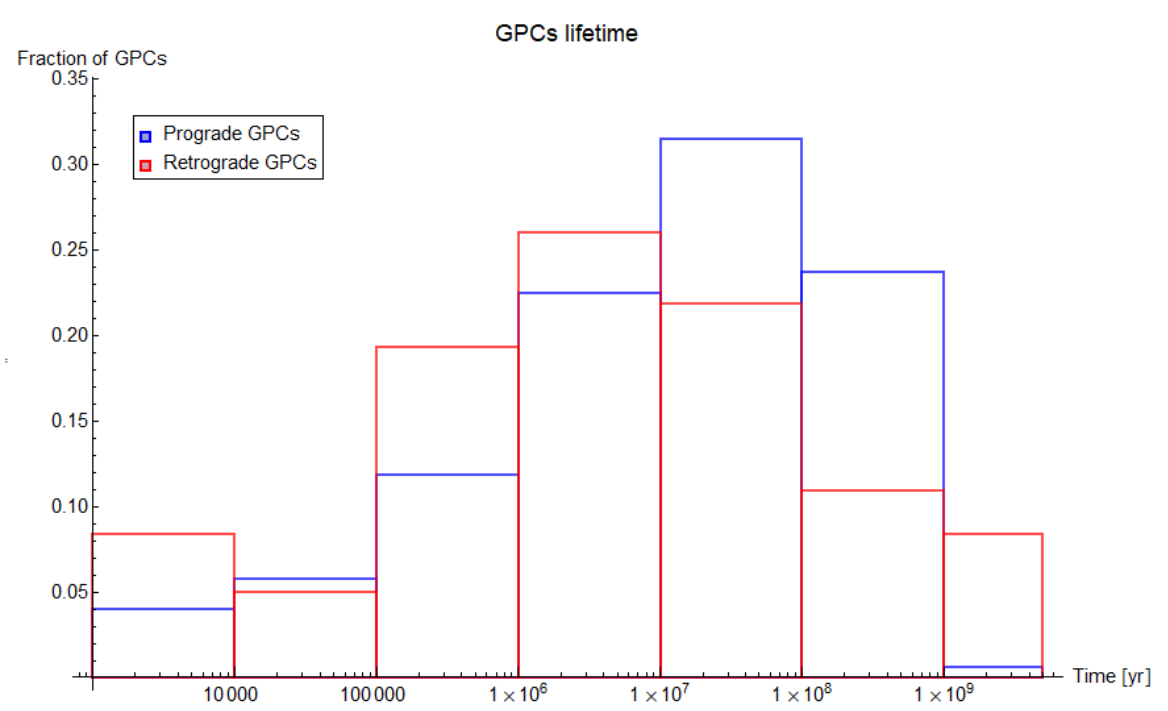

Fig. 2 Normalized distributions of the GPCs lifetime for both prograde and retrograde objects.

perihelion values with respect to those of the prograde population. The dynamical evolution of the two retrograde objects with high perihelion distances, i.e. the cases of 2011 KT19 and 2008 KV42, have been studied by Chen et al (2016) who obtained a mean dynamical lifetime of 500 Myr or greater and Gladman et al (2009) and Brasser et al (2012) who obtained a median lifetime of $200 \mathrm{Myr}$ for $2008 \mathrm{KV} 42$. However, Brasser et al (2012) claimed that the Oort cloud dominates over the SD as a source of the population with $i>70^{\circ}, 15$ au $<q<30$ au and $a<100 \mathrm{au}$, and found that those objects remain with their perihelion pinned to Uranus for a long time, showing short-period oscillations related to the Kozai mechanism. We found similar dynamical behaviors in our simulation for both long-lived objects.

Previous studies have been performed on the observed GPCs. Tiscareno and Malhotra (2003) explored the long term dynamical behavior of 53 known GPCs as of May 1st 2002 and followed their evolution for 100 Myr considering the perturbations of the four outer planets. They found that the median dynamical lifetime of their GPCs in their simulation was of 9 Myr and concluded that their sample was probably biased towards low eccentricities and low inclinations. Their sample of GPCs differed from ours, which covers a wider range of semimajor axis and inclination. In addition, our simulation lasts for $4.5 \mathrm{Gyr}$ while that of Tiscareno and Malhotra (2003) lasts for $100 \mathrm{Myr}$, at the end of which $20 \%$ of their sample have lifetimes exceeding $100 \mathrm{Myr}$. The combination of these changes in the simulation conditions could account for the difference we obtain in the median lifetime value. 
Tiscareno and Malhotra (2003) found that two thirds of their simulated particles were ejected from the Solar System during their simulation while one third was injected into the JFC population, in agreement with our simulation. They also found that GPCs did not stay in resonances for more than a few Myrs, in contrast with the SDOs behavior. In our simulation we find that some particles were captured in MMR for long periods of time. These type of captures, in MMR at high eccentricities and large semimajor axis values, are also found in SDOs. As mentioned, there are eight particles that survive for all the 4.5 Gyr in our simulation, six progrades and two retrogrades. The prograde particles that survived experienced captures in MMR for long periods of time that could be of the order of Gyrs and also resonance sticking. The two surviving retrogrades show a combination of temporary long-term resonance locking or resonance sticking moving from one MMR resonance to another and also Kozai resonances, and a conservation of perihelion distance in the zone between Saturn and Neptune but avoiding close encounters with the planets. The resonance sticking mechanism was first noticed by Duncan and Levison (1997) and since then is has been studied by a number of authors. In particular, it was found for objects in Neptune encountering orbits (scattered TNOs) (Lykawka and Mukai 2007; Yu et al 2018), and in the temporarily capture of Uranian and Neptune Trojans from the dynamical evolution of Centaurs (Alexandersen et al 2013). We note several resonance captures in the whole evolution with a tendency of GPCs to be captured into MMR with Neptune: 1:1, 3:2, 2:1 and 3:1 and 5:2. This can be noted in Figs. 3 and 4 where we plot the normalized time-weighted distribution for the GPC in the orbital element space. These maps show the normalized time fraction spent by the observed GPC across their evolution in different regions of the (a, e, i) space.

The blue zones are the most visited and thus, the ones were GPCs spend most of their time. Therefore, there is a dependence of the time of permanence on perihelion distances. The evolution near Neptune is slower, meaning slower diffusion timescales, and becomes faster close to Jupiter (see Fig. 3). In Fig. 4 the different dynamical evolution of prograde and retrograde GPCs can be seen. The densest zone (that of higher permanence) can be seen clearly for inclinations below $45^{\circ}$, while high inclination objects experience a faster evolution. However, there are some blue features visible in the retrograde population zone showing MMR captures as well.

\section{The GPC and Centaur populations from the Scattered Disk}

The giant planet region is a transitional zone which is mainly crossed by incoming TNOs, in particular those from the SD. In this paper we present new calculations about the current contribution of SDOs to the zone that comprises orbits with perihelion distances less than that of Neptune, i.e. the current population of giant planet crossers. Di Sisto and Brunini (2007) (DB07 in the following) built a model of an intrinsic current SD with real SDOs plus clones, which were numerically integrated for 4.5 Gyr to follow their dynamical 


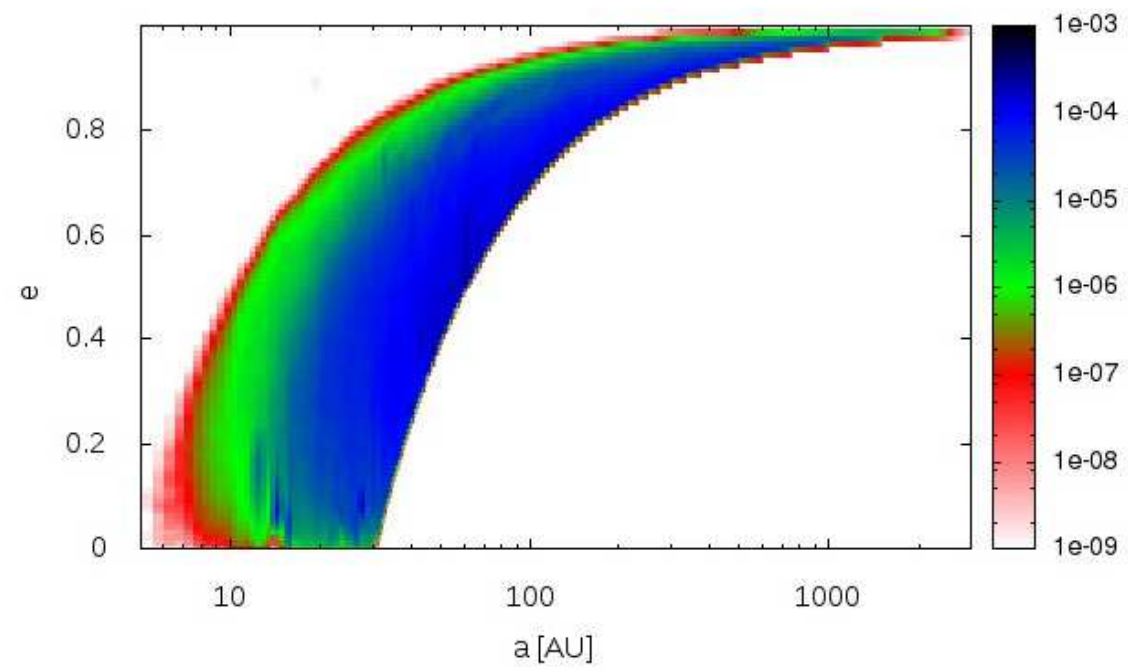

Fig. 3 Time-weighted distribution of the integrated GPC in the semimajor axis (a) vs. eccentricity (e) space.

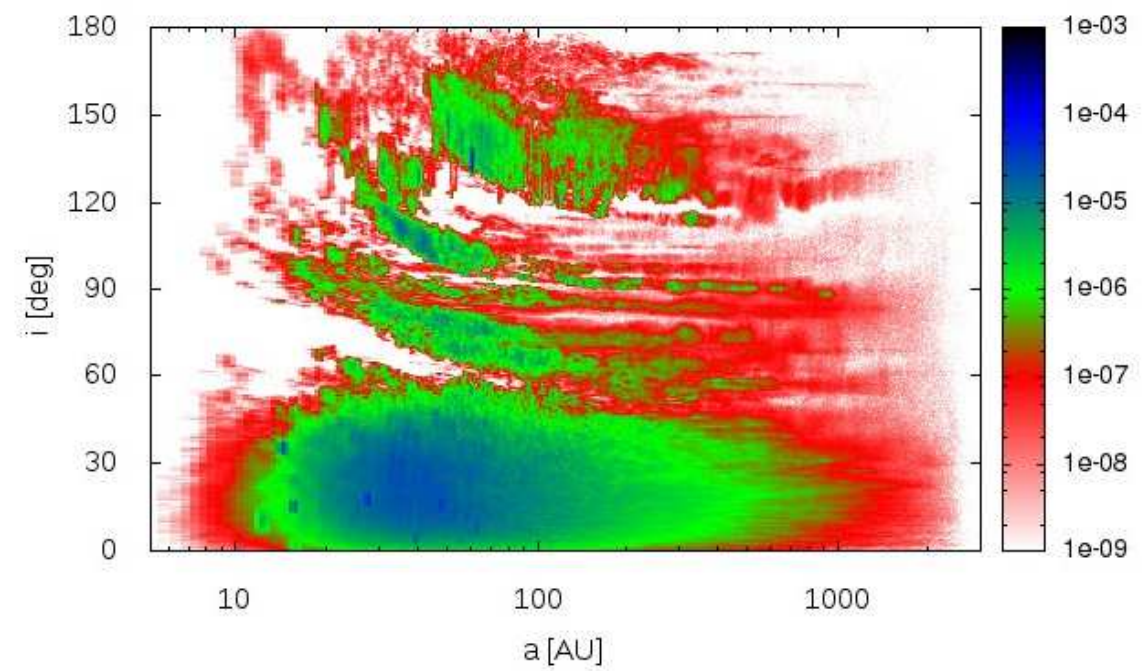

Fig. 4 Time-weighted distribution of the integrated GPC in the semimajor axis (a) vs. inclination (i) space.

evolution. They calculated the SDO contribution to the GPC zone $(q<30 \mathrm{au})$ in contrast to SDOs that have $q>30$. Di Sisto and Brunini (2007) obtained a rate of injection of SDOs to GPC of $5.2 \times 10^{-10} \mathrm{yr}^{-1}$ and a number of GPC 
with radius $R>1 \mathrm{~km}$ equal to $2.8 \times 10^{8}$. Volk and Malhotra (2008) also built a debiased model of the orbital distribution of the SD, and numerically integrated the particles for 4 Gyr. They defined the SDOs as those TNOs with $q>33$ au and $a>50$ au and found an escape rate of $1-2 \times 10^{-10}$ SDOs/yr, lower than DB07, mainly due to the difference between both debiased semimajor axis distributions. They also found that $10^{6}$ Centaurs with $D>1 \mathrm{~km}$ must exist in order to balance the loss of JFCs.

Since the SD is the main source of Centaurs and in light of the new observations and constraints on the size distribution of TNOs, we perform new numerical simulations following the model by DB07 but updated with the current observed SDO population.

\subsection{The model}

Since the model developed by DB07 in 2007, the number of observed SDOs has greatly increased. Di Sisto and Brunini (2007) recorded 95 observed SDOs from the Minor Planet Center database while now (up to April, 2019) this number has risen to 603. Those SDOs were defined as TNOs, which are not plutinos, with perihelion distances $30<q<39$ au, semimajor axis $a>40$ au and eccentricities $e>0.2$ to distinguish them from CTNOs (Elliot et al 2005). Those SDOs have absolute magnitudes in the range: $-1.1<H<9.8$, being (136199) Eris $(H=-1.1)$ the largest member with $D \sim 2500 \mathrm{~km}$ and 2015 PK312 $(H=9.8)$ the smallest observed object with $D \sim 50 \mathrm{~km}$.

DB07 developed a model of the current SDO population which accounts for the bias in the discovery probability for different semimajor axis (based on Fernández et al (2004)) since SDOs can be discovered when they are close to their perihelia. Therefore, they found that an intrinsic semimajor axis distribution of SDOs would be given by:

$$
f(a) \propto a^{-2}
$$

DB07 also considered the bias towards low inclination discoveries by existing surveys, and proposed a Brown intrinsic inclination distribution (Brown 2001) given by:

$$
F(i) d i \propto \sin i \exp ^{\frac{-i^{2}}{2 \sigma_{i}^{2}}}
$$

where $\sigma_{i}=12^{\circ}$ from the model developed by Morbidelli et al (2004). From observational surveys, recent works have found different values of $\sigma_{i}$. Gulbis et al (2010) analyzed the inclination distributions of the different TN populations based on data from the Deep Ecliptic Survey. They found that for the scattered population, the general inclination distribution form is consistent with a Brown distribution with $\sigma_{i}=6.9^{\circ}$. Petit et al (2017) study the High Ecliptic Latitude extension (HiLat) of the CanadaFrance Ecliptic Plane Survey (CFEPS) and found that for other populations than the classical one, the combined CFEPS + HiLat sample allows an inclination distribution with 
$12.5^{\circ}<\sigma_{i}<20^{\circ}$. Therefore, although there is disparity in the survey $\sigma_{i}$ values, the adopted value of $\sigma_{i}=12^{\circ}$ seems to be consistent with the observations.

Our first test for the present review was to study if considering the new observations, the orbital distributions of observed objects approaches the intrinsic distribution proposed in DB07 model. This comparison can be seen in Fig. 5. As shown in the figures, the current observations seem to follow the
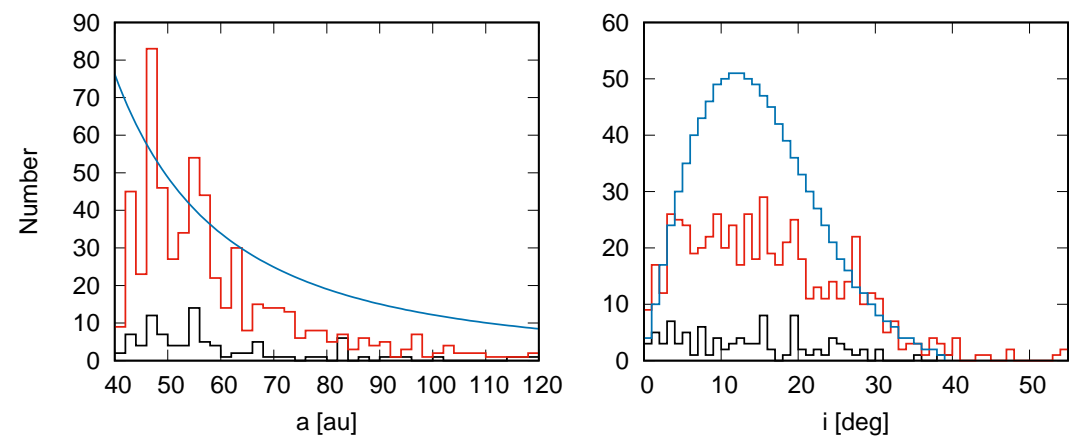

Fig. 5 Intrinsic orbital element distribution by DB07 (blue line), Observations used in DB07 model (black line), observations up to April, 2019 (red line).

same trend as the debiased semimajor axis and inclination distributions. In the updated observations, there are some objects with higher inclinations and others at larger distances than the observations used in DB07, but they account for a small fraction of the total number of objects. This analysis encouraged us to perform new numerical simulations with the model used in DB07 but considering all the updated observations. Therefore, in the present work we use Eqs. (11) and (2) to build the model as we explain in the next section.

\subsection{The numerical simulation}

As mentioned, our current observed population of SDOs $(30<q<39$ au, $a>40$ au and $e>0.2$ ) is of 603 objects, up to April, 2019. They are plotted in Fig. 6.

With those real SDOs (603) we generate 5167 clones so that the total of the initial particles (5770), that is to say real plus clones, fit the distribution of semimajor axis (given by Eq. (1)) and inclination (given by Eq. (2)) of the model. We proceed in the same way that in DB07. We first generate the number of clones for each value of semimajor axis, then change the semimajor axis of almost half of the clones (selected randomly from the total sample of synthetic SDOs) by $\delta$ such that $-2 \times 10^{-4}<\delta<2 \times 10^{-4}$ and allocate the mean anomaly for all the clones randomly between $0^{\circ}$ and $360^{\circ}$. Then, we assign random inclinations for the clones in such a way that the total number of particles (real plus clones) fit the proposed Brown distribution (Eq2). 

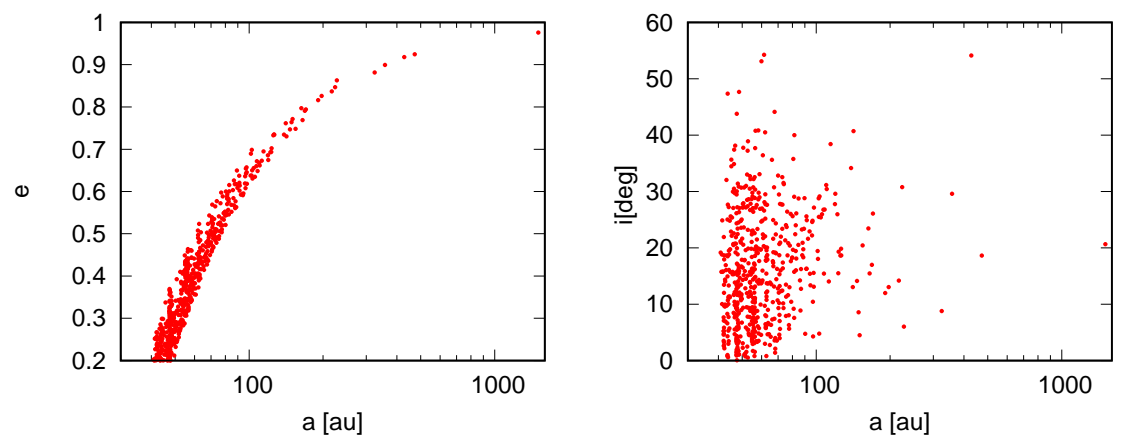

Fig. 6 Semimajor axis vs eccentricity and Semimajor axis vs inclination of the real SDOs by April 2019.

Therefore, we performed a numerical integration of 5770 massless particles under the gravitational influence of the Sun (including the masses of the terrestrial planets), the four giant planets and Pluto, with the hybrid integrator EVORB (Fernández et al 2002). Pluto was only included in the simulation for the purpose of a future work. Its effect on the dynamical evolution of SDOs and their link to GPC and Centaurs is negligible as was previously noted by Nesvorný et al (2000); Tiscareno and Malhotra (2009); even the effects of the largest TNOs are minor in the supplying of Centaurs and JFCs (Muñoz-Gutiérrez et al 2019).

The integration step was 0.2 years and the total time span, 4.5 Gyr. The code cutting conditions were: collision with a planet, reaching a semimajor axis $a>5,000$ au (ejection), or a distance $r<5.2$ AU, i.e. the zone of Jupiter Family Comets (JFC) where the terrestrial planets perturbations are needed to account for a real dynamical evolution and a physical model is also required (Di Sisto et al 2009).

\subsection{Results}

The general results of the new simulation are similar to that of DB07. However, in the new simulation we include a larger number of real objects which extent to larger $a$ and $i$ values, the integration step is somewhat smaller than in the previous integration and we include Pluto as another perturbing massive object. There are also new estimations of the number and size distribution of SDOs. Therefore, we update the results regarding the contribution of SDOs to the GPC population and also evaluate their contribution to Centaurs.

From the initial 5770 particles, $18(0.3 \%)$ collide with a planet: 4 with Saturn, 4 with Uranus and 10 with Neptune. 3801 particles $(50.6 \%)$ reach $a>5000 \mathrm{au}, 884(15.3 \%)$ reach the zone of $r<5.2 \mathrm{AU}$, and the remaining $1951(33.8 \%)$ survive as a SDO. These percentages are a little different from DB07; in particular the number of objects that enter the JFC zone is lower while the number of surviving particles is higher. This is due to the fact that 
in the present model we extend the SD up to much higher distances. Thus, the evolution of those particles is slower, having less chances of encountering Neptune, and then tend to remain longer in the SD. On the other hand, as was found by DB07, the delivery to the JFC region is mainly from regions with small perihelion distances and small semimajor axes.

We computed the encounters between a particle and a planet if the particle reached a distance to the planet within their Hill radii. From the total number of encounters, $4.9 \%$ are with Pluto, $73.4 \%$ with Neptune, $17.8 \%$ with Uranus, $3.8 \%$ with Saturn and $0.1 \%$ with Jupiter. From the total number of particles in the integration, almost all, i.e. $92 \%$ encounter Pluto, $46 \%$ encounter Neptune, $38 \%$ encounter Uranus, $28 \%$ Saturn and $4 \%$ Jupiter. However, the encounters with Jupiter and, in particular, the number of particles at encounter, is sub-assessed due to the cutting condition at $r=5.2 \mathrm{au}$. It has to be noted that, although the majority of the particles encounter Pluto, the real proportion of encounters with Pluto in the whole simulation (12 per particle) is much smaller than that with Neptune (358 per particle) which is the planet that actually scatters the SDOs towards the GPC and Centaurs region. The encounter statistics give a mean probability of encounters inside the Hill radius per object per year of $2.7 \times 10^{-9}$ for Pluto and $8 \times 10^{-8}$ for Neptune, more than an order of magnitude larger than for Pluto. Those numbers are in agreement with the probability calculated by the Opik method. Therefore, our simulation confirms that Pluto has a minimal effect on the supply of GPC, Centaurs and therefore JFCs, as was previously stated (Nesvorný et al 2000; Tiscareno and Malhotra 2009).

From the initial SDOs in the integration, $70 \%$ are delivered to the GPC zone. The mean lifetime $\left(l_{\mathrm{GPC}}\right)$ there is $68 \mathrm{Myr}$, the most likely value being between 10 and 100 Myr as was found in DB07 (see Fig. 4). There is a strong dependence of the lifetime as a GPC with the initial inclinations in the SD as can be seen in Fig. 7. It is worth noting that SDOs with initial inclinations between 50 and 55 degrees have large mean lifetimes in the GPC zone. However from the Brown distribution they are a few number of particles. Also, we notice the same heavy reliance of mean lifetime of GPC with the perihelion distance as DB07 (see their fig. 6), the mean lifetime being larger for greater q values.

From the simulation we estimate the injection rate of GPC from the SD. We recorded every 1,000 years, the orbital elements of SDOs when they have $q<30$ AU. From this file, we compute the first time a SDO enters this zone as the time of injection. Therefore, we can analyze the time dependence of the quotient between the cumulative number of SDOs that enter the GPC zone $\left(N_{G P C}\right)$ and the number of SDOs that remain in the SD $\left(N_{S D O}\right)$. This relation is plotted in Fig. 8 and is well fitted by the linear relation whose slope is:

$$
d\left[N_{G P C} / N_{S D O}\right] / d t=Y
$$

where $Y=4.025 \pm 0.008 \times 10^{-10} N_{S D O} /$ year is the rate of injection of GPC from the SD. This linear fitting implies that if we know the current number of SDOs, the present cumulative number of GPCs $\left(N_{G P C}(>D)\right)$ coming from 


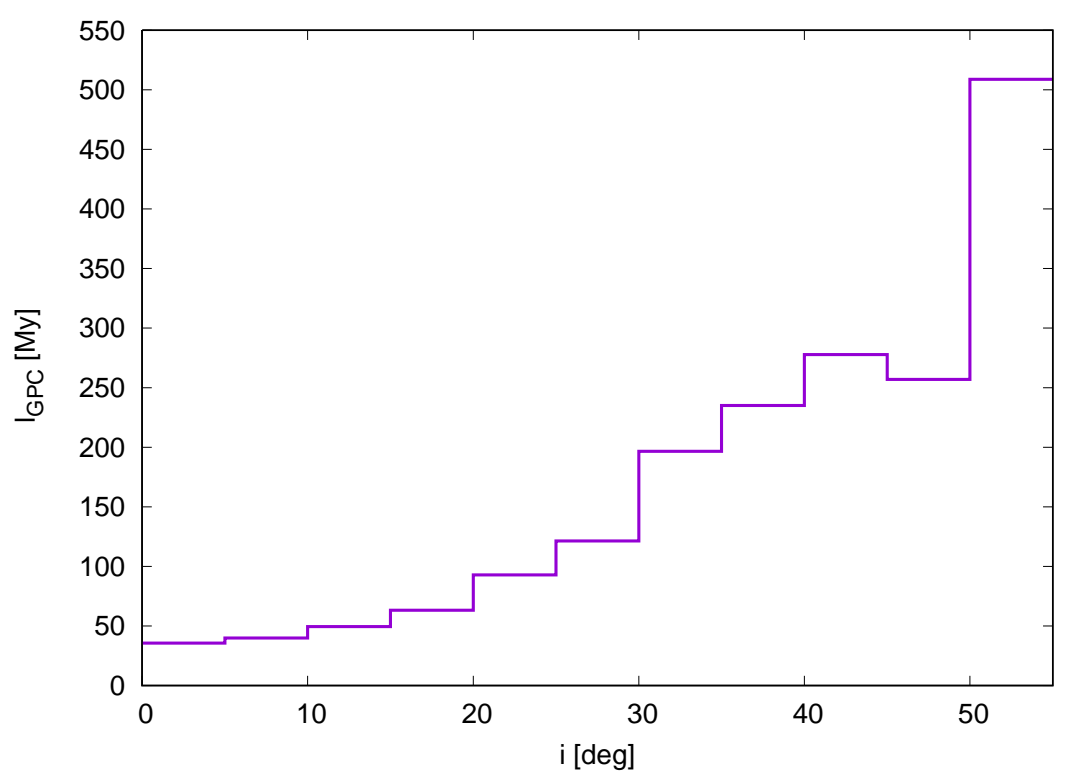

Fig. 7 Mean lifetime as a GPC vs initial inclination.

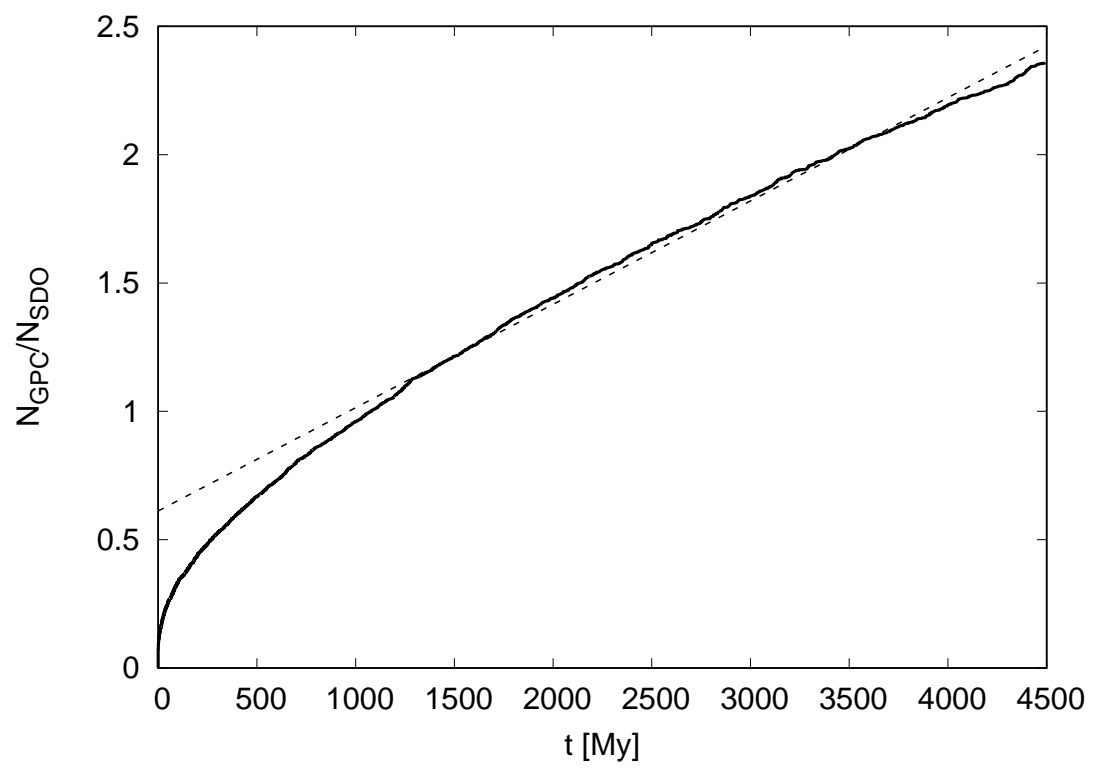

Fig. 8 Number of GPC $\left(N_{G P C}\right)$ coming from the SD with respect to the surviving SDOs $\left(N_{S D O}\right)$ as a function of time (solid line). The dashed line represents the linear fit to data (see text).

the SD would be given by:

$$
N_{G P C}(>D)=Y N_{S D O}(>D) l_{G P C},
$$


where $N_{S D O}(>D)$ is the current population of the SD greater than a diameter $D$, and the rate of injection will be $Y N_{S D O}(>D) /$ year. There are various estimations of the SD population from different surveys. Di Sisto and Brunini (2011) analyzed the number and size-frequency distribution (SFD) of SDOs based on the works of Parker and Kavelaars (2010b,a). They adopted a broken power-law size distribution with a differential index of large objects given by $s_{1}=4.7$ (Elliot et al 2005), a break at diameters $d \sim 60 \mathrm{~km}$ and two limit values for the differential index $s_{2}=2.5$ and 3.5 for $d<60 \mathrm{~km}$ given the uncertainty of the SFD for small objects (Bernstein et al 2004; Gil-Hutton et al 2009; Fraser and Kavelaars 2009; Fuentes and Holman 2008; Fuentes et al 2009). However, the recent discoveries by OSSOS increased the number of small SDOs and Centaurs and allowed for new estimations of the SFD and number of objects. Lawler et al (2018b) found that a break in the SFD is required at $D \sim 100 \mathrm{~km}$. They found a faint-end slope of the magnitude size distribution $\alpha=0.4-0.5$ which corresponds to a differential size index of $s=3-3.5$. Therefore, we propose here the same SFD of SDOs as in Di Sisto and Brunini (2011) but with the break at $D=100 \mathrm{~km}$. Also, we consider the differential index for $D<100 \mathrm{~km}$ for three values $s_{2}=2.5,3$ and 3.5 . Thus, the cumulative number of SDOs will be given by:

$$
\begin{array}{lll}
N(>D)=C_{0}\left(\frac{1 \mathrm{~km}}{D}\right)^{s_{2}-1} & \text { for } & D \leq 100 \mathrm{~km}, \\
N(>D)=3.5 \times 10^{5}\left(\frac{100 \mathrm{~km}}{D}\right)^{s_{1}-1} & \text { for } & D>100 \mathrm{~km},
\end{array}
$$

where $C_{0}=3.5 \times 10^{5} \times 100^{s_{2}-1}$ by continuity for $D=100 \mathrm{~km}$.

This relation is plotted in Fig. 13. We obtained for the intermediate index $s_{2}=3$ that $N(>2 \mathrm{~km})=8.75 \times 10^{8}$ and $N(>10 \mathrm{~km})=3.5 \times 10^{7}$, while Nesvorný et al (2017) obtained $N(>2 \mathrm{~km})=4.4 \times 10^{8}$ and $N(>10 \mathrm{~km})=$ $1.5 \times 10^{7}$ for the inner SD $(50<a<200 \mathrm{au}$, i.e. a part of the complete SD) by calibrating the source population of the observed ecliptic comets through a dynamical model. Both estimations are in agreement with ours within a factor of $\sim 2$, which given the slightly different population ranges is considered acceptable.

It has to be noted that it has been inferred from cratering counts on the Pluto and Charon system (Singer et al 2019) and from cratering studies on the satellites of the outer planets (Kirchoff and Schenk 2010; Di Sisto and Zanardi 2013; Bierhaus and Dones 2015) that there may be an additional break in the SFD slopes of TNOs below 1-2 km in diameter. A series of geological studies in relation to impact cratering has been performed particularly on the Pluto system (Moore et al 2016; Robbins et al 2018). A discussion about how geologic processes affect the crater size distribution was carried out by Singer et al (2019). However, a general study about geological processes that could erode small craters on all the objects which are the targets of collisions by Centaurs would be recommendable before asserting the existence of a new break. Therefore, we are not going to consider this range of sizes in the following. 
The cumulative number of GPC $\left(N_{G P C}(>D)\right)$ can be calculated from Eqs. (41) and (5). For example, we have $9560 \mathrm{GPC}$ with $D>100 \mathrm{~km}$ and using the intermediate index $s_{2}=3$ for the SFD of SDOs for small objects $N_{G P C}(>$ $10 \mathrm{~km}) \sim 2.3 \times 10^{6}$ and $N_{G P C}(>1 \mathrm{~km}) \sim 2.23 \times 10^{8}$, a little less than what was found by DB07. The rate of injection, calculated by $Y N_{S D O}(>D) /$ year yields for example, 3 SDOs with $D>1 \mathrm{~km}$ every 2 years or 14 SDOs with $D>10 \mathrm{~km}$ every 100 years.

From our numerical integration it is possible to calculate the normalized time-weighted distribution for the GPC in the orbital element space. We divide the $(\mathrm{a}, \mathrm{e}, \mathrm{i})$ space in bins of size $\delta a=0.2 \mathrm{au}, \delta e=0.01$ and $\delta i=0.2^{\circ}$ and calculate the normalized time fraction spent by GPCs in different regions of the (a, e, i) space. These calculations are plotted in Figs. 9 and 10. Those plots form dynamical maps of permanence and thus represent our modeled GPC distribution, assuming time-invariability. The observed GPC are also plotted. The color code is indicative of the fraction of time spent in each zone (blue for most visited regions, red for least visited). As in DB07, the orbital element region with greater probability of occupation is the one with $\sim 20 \lesssim a \lesssim 80$ au, and $i \lesssim 30^{\circ}$. In Fig. 10 there are various blue vertical features that correspond to mean motion resonances, being the $2: 1$ at $a \sim 48$ au, corresponding to the Twotinos, the most populated one. Some of those features correspond to objects that are initially in a MMR, however they are a very small fraction of the whole set. We detected captures on several MMR with Neptune, Uranus and Saturn being the most frequent the 1:1, 2:1, 5:2, 3:1 MMR with Neptune and the 2:3 MMR with Uranus.

The observed GPC are in the zone also comprised by our model. However, there are high inclination objects and retrograde ones (not plotted in Fig. 10) that cannot be explained by our model. Therefore, the source of retrograde GPCs is not the SD but the Oort cloud as proposed by other studies (e.g. Volk and Malhotra (2013); Brasser et al (2012); Nesvorný et al (2017); Nesvorny et al (2019)). From Fig. 9, the zone of perihelion distances between 20 and 30 au must be densely populated, according to our model. In addition, we note that the diffusion time scale is short for objects that reach perihelion distances within that of Saturn, in comparison to those that lie farther.

However, the instability of the region near the orbit of Jupiter is a byproduct of the boundary conditions of our model, which is not valid inside the region delimited by this planet.

The dynamical evolution in the Giant planetary zone is strongly dependent on the perihelion distance, as is the mean lifetime in that zone. Therefore, the GPC are not uniformly distributed with respect to $q$. This can be seen in Fig. 11] where we plot the normalized time-weighted distribution of perihelion distances for the GPCs of our model. Rescaling this distribution by $N_{G P C}(>$ $D)$, obtained from Eqs. (4) and (5) it is possible to obtain the number of GPCs for each perihelion value. We note that the number of GPC grows exponentially with perihelion, being the region near Neptune the most populated one. This is connected with their origin as particles scattered by this planet. 


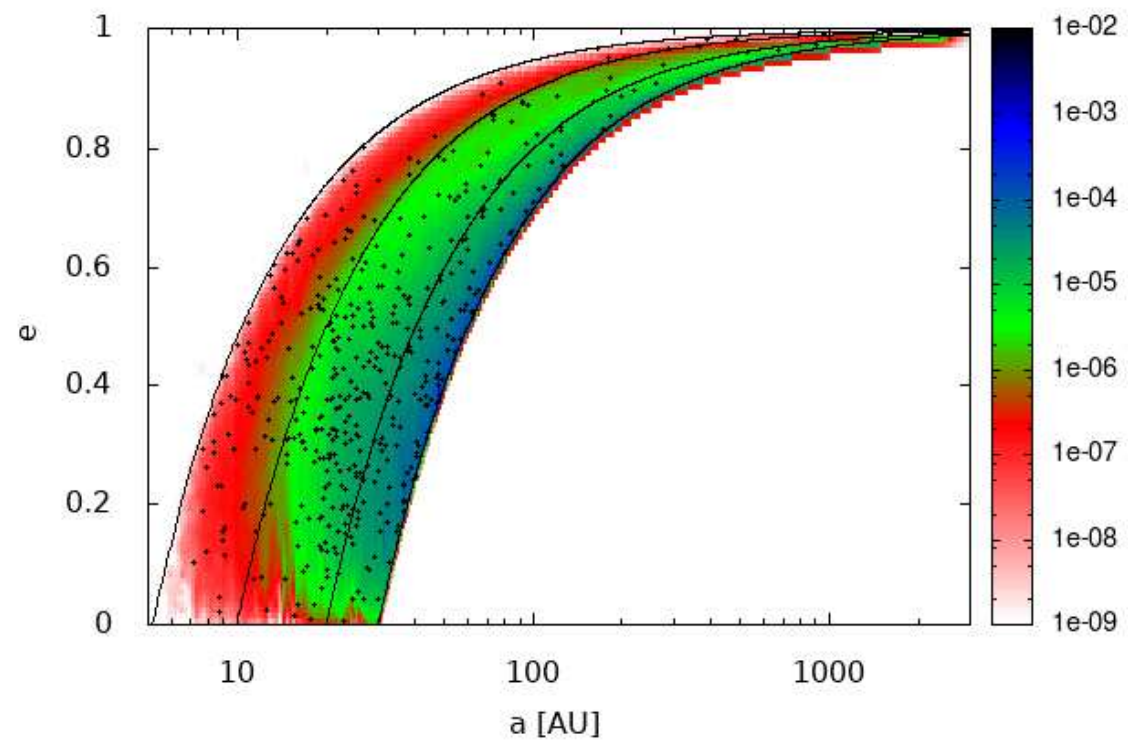

Fig. 9 Time-weighted distribution of the GPC obtained from the simulation in the semimajor axis (a) vs. eccentricity (e) space. Black points represent the observed population.

\subsection{The contribution to Centaurs}

In the region restricted only by semimajor axes between $5.2<a<30$ au, the minor bodies are the Centaurs. There are observational results on the number and size distribution of Centaurs which can be used to compare them with our model. Although Centaurs are in general restricted to $5<a<30$ au, our restriction on $a>5.2$ au doesn't affect the time-averaged statistics, since the dynamical timescales get very short near Jupiter, as mentioned.

Thus, we proceed as in the previous subsection, but considering the contribution of SDOs to Centaurs. 34\% of SDOs enter the zone of $a<30 \mathrm{au}$, and we extract from the output orbit file, the first time a SDO enters this zone. We calculate the cumulative number of Centaurs $N_{C}$ coming from the $\mathrm{SD}$ in relation to the surviving number of SDOs $\left(N_{S D O}\right)$ for each time. In this case, some SDOs left the integration, never entering the Centaur zone, and then we have to extract them from the remaining population at each time step. This relation is plotted in Fig. 12 where we can see the linear fitting to the data. The slope of this line is the rate of injection of Centaurs from the SD, $Y_{C}=1.796 \pm 0.005 \times 10^{-10} N_{S D O} /$ year. The mean lifetime in the Centaur zone, calculated as before, is $l_{C}=7.2 \mathrm{Myr}$. Therefore, the cumulative number of Centaurs $\left(N_{C}(>D)\right)$ from our model can be obtained from Eqs. (4) and (5) but considering $Y_{C}$, and $l_{C}$ (instead of $Y_{G P C}$, and $l_{G P C}$ ). 


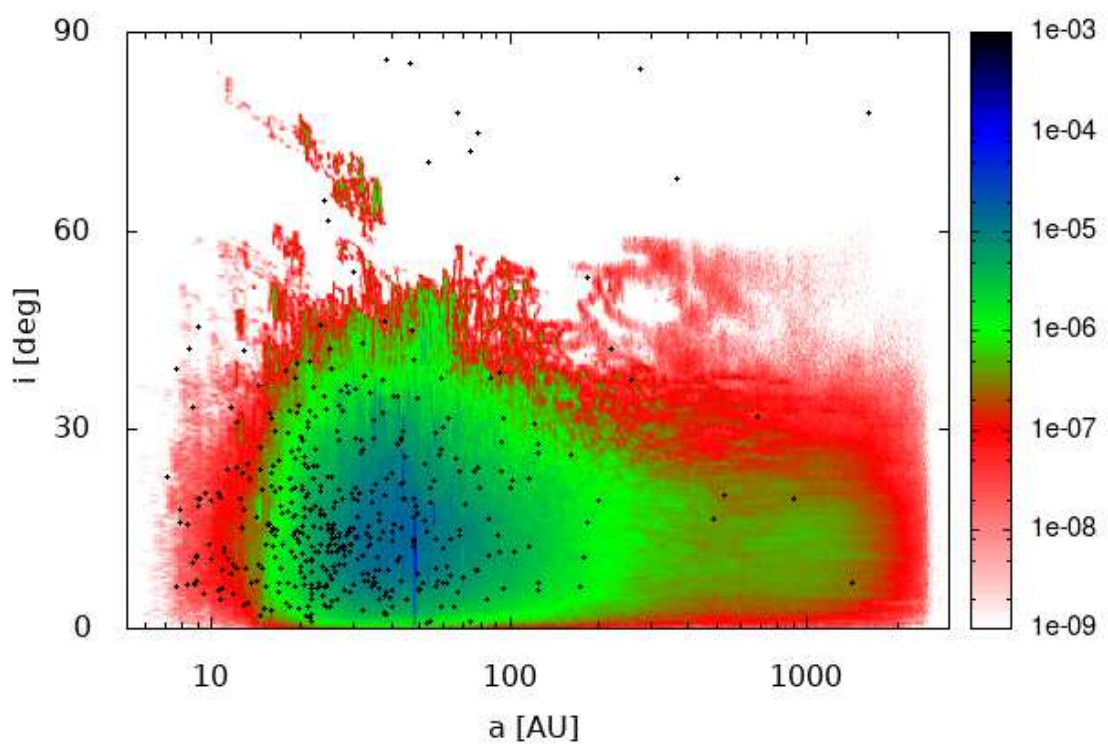

Fig. 10 Time-weighted distribution of the GPC obtained in the simulation in the semimajor axis (a) vs. inclination (i) space. Black points represent the observed population.

From those calculations we plot in Fig. 13, the cumulative number of SDOs $\left(N_{S D O}(>D)\right)$, the cumulative number of Centaurs $\left(N_{C}(>D)\right)$ and two estimations by Lawler et al (2018b) and Nesvorny et al (2019). Our model predicts a somewhat greater number of large Centaurs than Lawler et al (2018b) and Nesvorny et al (2019), but the numbers for $D<100 \mathrm{~km}$ are similar and lie between the error bars. Nesvorny et al (2019) calibrated the Centaur population from Jupiter Trojans and predicted a population of Centaurs with a size distribution with a differential size index equal to 3.1 for $5<D<100$ $\mathrm{km}$, i.e. between our results for $s_{2}=3$ and $s_{2}=3.5$.

\subsection{Dynamical evolution}

The dynamical evolution in the GPC zone was analyzed from our new numerical simulation. Our results show that the particles that achieve the largest lifetimes exhibit essentially four types of dynamical behaviors, as presented in DB07. These are:

- Between the orbits of Saturn and Neptune, particles that present slight variations of $q$ throughout their whole lifetime, together with a nearly constant perihelion longitude, eccentricities exceeding $\sim 0.8$ and large semi- 


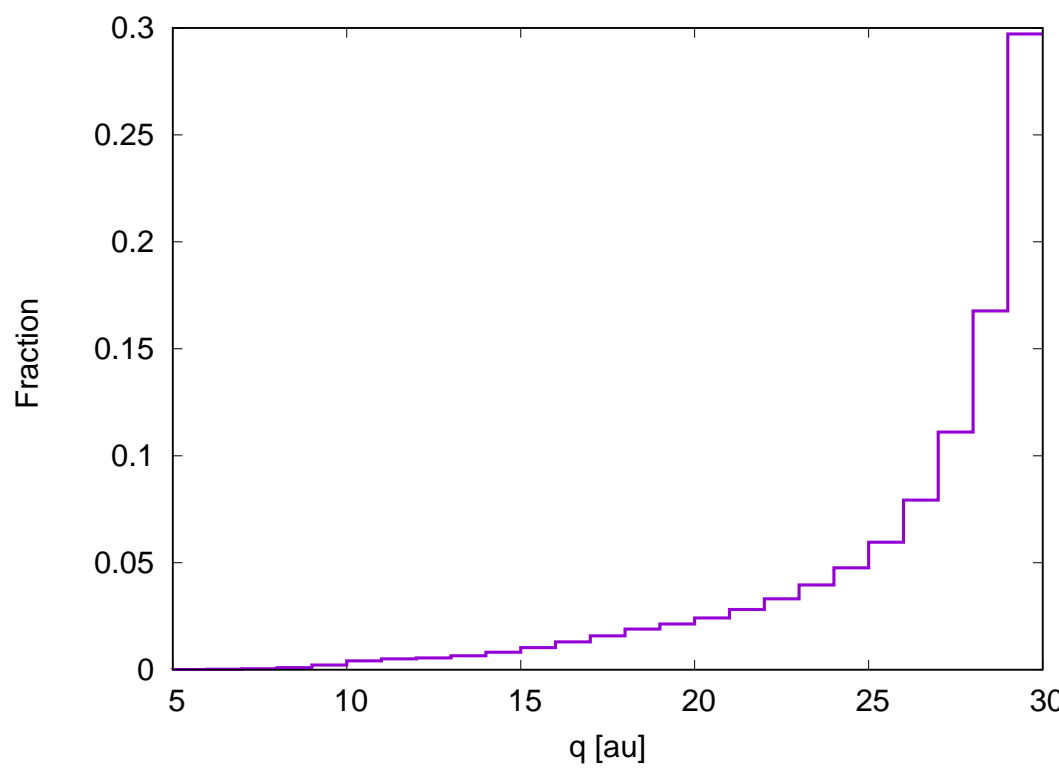

Fig. 11 Normalized time-weighted distribution of the GPC obtained in the simulation with respect to perihelion distances $q$.

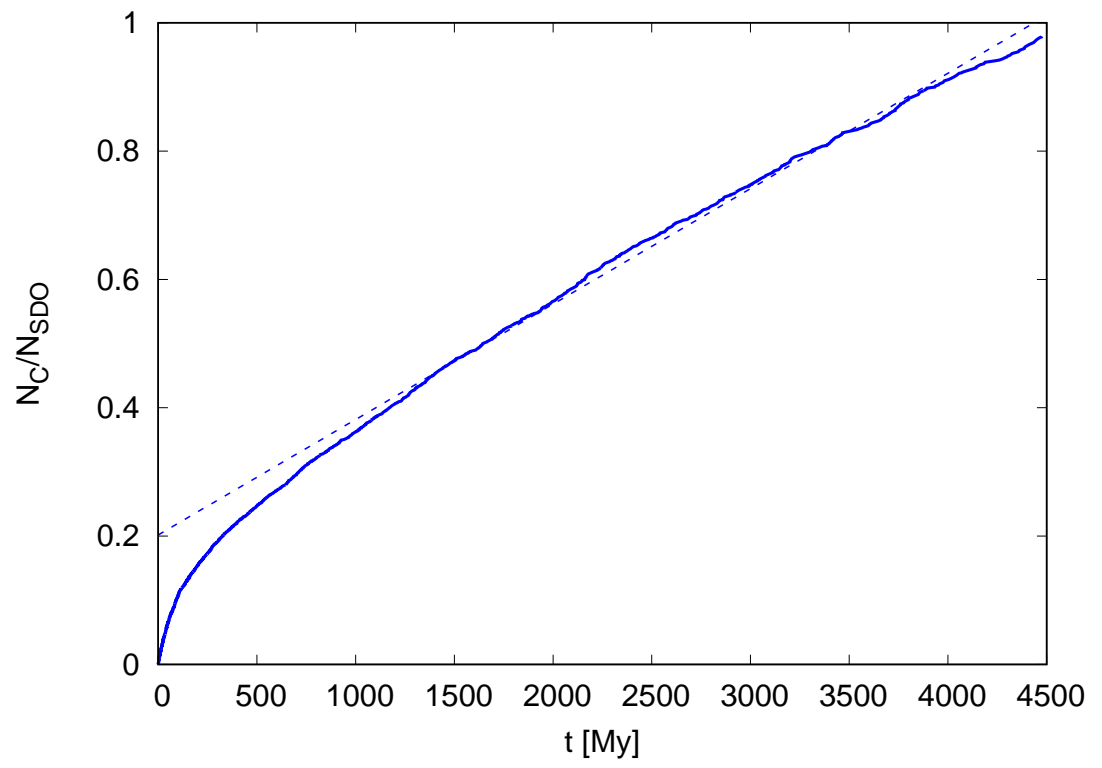

Fig. 12 Cumulative number of Centaurs $\left(N_{C}\right)$ coming from the SD with respect to the surviving SDOs $\left(N_{S D O}\right)$ as a function of time (solid line). The dashed line represents the fit to data (see text). 


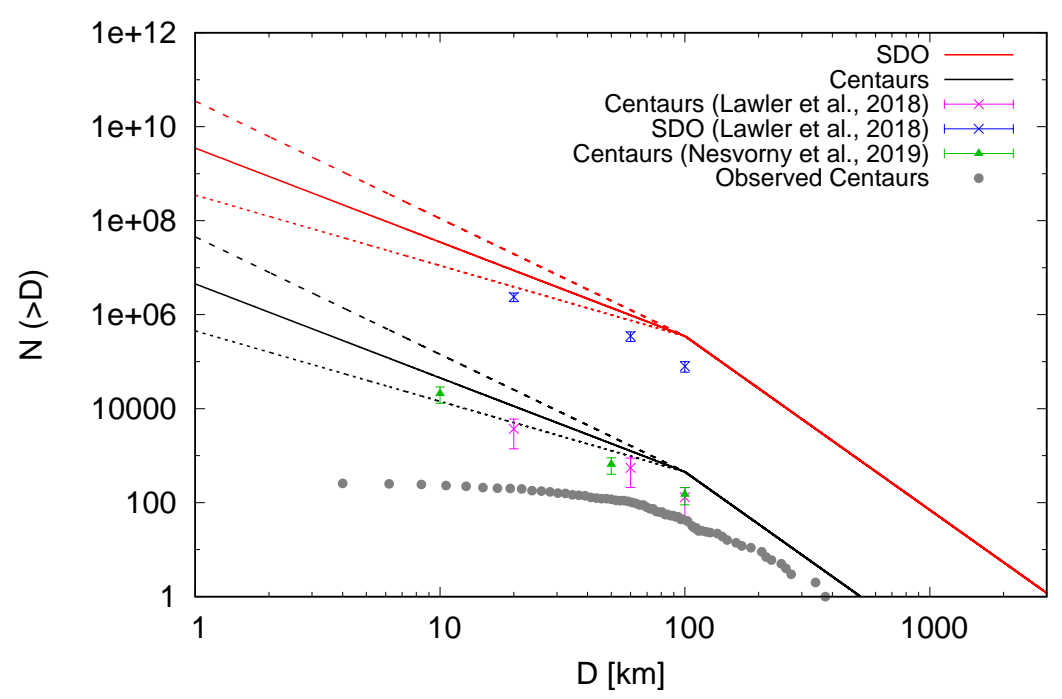

Fig. 13 Cumulative number of SDOs $\left(N_{S D O}(>D)\right)$ obtained from Eq. (5) and cumulative number of Centaurs $\left(N_{C}(>D)\right)$ from our model. Gray points correspond to the observed Centaur population, green points represent the number of Centaurs estimated by Nesvorny et al (2019) and magenta points correspond to estimations of Lawler et al (2018b). Blue points represent the number of SDOs estimated by Lawler et al (2018b). For the observed population and survey estimations we use an albedo $p_{v}=0.06$ to convert absolute magnitude to diameter.

major axes ( $>100 \mathrm{au}$ ). This behavior tends to stabilize the orbit and its orientation, minimizing the number of encounters.

- Transfers between mean motion resonances, known as resonance sticking, in the zone of $\sim 30$ au $<a \lesssim 200$ au. There are also transfers between MMR and Kozai resonances.

- For large values of the semimajor axis $(a \gtrsim 200 \mathrm{au})$ the general dynamical evolution is a low increase of semimajor axis but keeping the perihelion distances near Neptune. Thus, the objects are continuously entering and leaving the GPC zone up to ejection (injection to the GPC zone is more rare).

- GPC that enter a MMR or Kozai resonance and remain there up to the end of the integration.

Those mechanisms are responsible of the long dynamical lifetime between $5.2<q<30$ au.

In particular, Lykawka and Mukai (2007) found that evolution of scattered TNOs is described by multiple temporary resonance sticking and continuous scattering by Neptune and that this mechanism is relevant mostly at $a<$ $250 \mathrm{au}$, consistent with our result. Yu et al (2018) predicted that the current transient-sticking population comprises $40 \%$ of the total transiently stuck + scattering TNOs, and therefore is a very important mechanism in the SD zone. 
However, the general path through this region is a "hand off" from the gravitational control of one planet to another as was already found by Levison and Duncan (1997). Once a particle reaches a region near Jupiter and experiences close encounters with this planet, it rapidly suffers either an ejection or an injection into the inner region of the Solar System. This is connected to the correlations of mean lifetime with perihelion distance already mentioned.

Bailey and Malhotra (2009) found that the long-term orbital evolution of Centaurs exhibit two types of behavior that are strongly correlated with Centaur lifetime. Centaurs with shorter lifetimes are characterized by diffusive evolution of semimajor axis and the ones with longer dynamical lifetimes are dominated by resonance hopping. Those mechanisms were also reported by Tiscareno and Malhotra (2003) and our previous work DB07.

\section{GPC and Centaurs from other sources}

The zone of $q<30$ au is also fed by other minor body populations. Here we review the works about other contributions to that zone and compare them with the main contribution from the SD.

\subsection{From Plutinos and other TN MMR}

The 2 : 3 MMR with Neptune, i.e. the Plutinos is the most populated observed resonance in the TN region. Morbidelli (1997) analyzed the dynamical structure of the $2: 3 \mathrm{MMR}$ and found that there exists a slow chaotic diffusion zone that should be an active source of Neptune-encountering bodies at current epoch of the Solar System and then a source of current Centaurs and JFCs. However, they found that only $10 \%$ of the Plutinos in this weakly chaotic zone have been delivered to Neptune-encountering orbits in the last Gyr. Tiscareno and Malhotra (2009) performed numerical integrations over 1 Gyr timescale on objects in the $2: 3$ and $1: 2$ MMR with Neptune. They found that the escaped Plutinos and Twotinos spend roughly equal amounts of time as Centaurs as they do as SDOs. However, only $\sim 20 \%$ of both resonant objects survived the 1-Gyr integration, and only $\sim 15 \%$ are projected to survive for 4 Gyr. Thus, although the rate of escape should be high at initial times, it would be low during the present epoch in agreement with Morbidelli (1997).

The collisional evolution of resonant objects could be another way of removal from the resonance; however, de Elía et al (2008) found a flux rate of escape of $0.5 \%$ of Plutinos in $10 \mathrm{Gyr}$, which is much less than the dynamical removal. Di Sisto et al (2010) analyzed the contribution of Plutinos to the GPC zone and found that almost all the Plutinos that escape from the resonance enter the GPC zone with a current rate of injection of $1.62 \times 10^{-10} \mathrm{yr}^{-1}$, i.e., $\sim 3$ times less than the injection from the SD found by DB07. They considered the number and size distribution of Plutinos proposed by de Elía et al (2008) 
which is given by:

$$
\begin{array}{lll}
N_{P}(>D)=C\left(\frac{1 \mathrm{~km}}{D}\right)^{p} & \text { for } & D \leq 60 \mathrm{~km}, \\
N_{P}(>D)=7.8 \times 10^{9}\left(\frac{1 \mathrm{~km}}{D}\right)^{3} & \text { for } & D>60 \mathrm{~km},
\end{array}
$$

where $C=7.9 \times 10^{9} \times 60^{p-3}$ by continuity for $D=60 \mathrm{~km}$ and two values are considered for the cumulative power-law index $p: 2.5$ and 1.5 .

Alexandersen et al (2016) performed a detailed study and analysis of the Plutino size distribution and found the cumulative number of Plutinos to be $N_{P}(H<8.66)=9000 \pm 3000$ and $N_{P}(H<10)=35000 \pm 10000$ while from Eq. (6), considering an albedo of 0.06 , the corresponding numbers in terms of diameter are $N_{P}(D>100 \mathrm{~km})=7900$, and $N_{P}(D>60 \mathrm{~km})=36500$, both in perfect agreement with Alexandersen et al (2016). Therefore, Di Sisto et al (2010) calculated from the Plutino SFD and the rate of injection to GPC that there could be between $1.8 \times 10^{6}$ and $1.8 \times 10^{7}$ GPC with $D>1 \mathrm{~km}$ from Plutinos in the current population. This is an order of magnitude less than that from the SD. This contribution together with that of the SD and from Jupiter Trojans can be seen in Fig. 14. Using the simulation from Di Sisto et al (2010) it is possible to compute the rate of injection of Plutinos to the Centaur zone $(a<30 \mathrm{au})$. We find that $80 \%$ of the Plutinos that escape from the resonance reach the Centaur zone. Extracting from the output orbit files the first time a Plutino enters this zone, we obtain that their rate of injection is of $Y_{p}=1.316 \pm 0.002 \times 10^{-10} N_{P} /$ year. Muñoz-Gutiérrez et al (2019) obtained a rate of injection from the TN resonant population to the JFCs zone (defined by $2<T<3$ and $q<2.5 \mathrm{au}$ ). They found that the resonant regions that contribute mainly to JFCs are the 3:2 and 5:2 MMRs and the total rate of injection to JFCs is of $Y_{p}=1.07 \times 10^{-10} \mathrm{~N} /$ year by including as massive particles 34 large TNOs and $Y_{p}=0.954 \times 10^{-10} \mathrm{~N} /$ year without those large TNOs. Both Muñoz-Gutiérrez et al (2019) rates are very similar and slightly smaller than our rate since they are calculated for injection to JFCs an our calculation is for Centaurs. We also calculate the Plutino mean lifetime as a Centaur to be $l_{P C}=8.8 \mathrm{Myr}$. Therefore, proceeding in the same way as for SDOs (see Eq. (4)), but considering the injection rate and mean lifetime for Plutinos to Centaurs and the number of Plutinos from Eq. (6), we calculate the cumulative number of Plutinos in the Centaur population. This contribution together with that of the SD and from Jupiter Trojans can be seen in Fig. 15.

Di Sisto et al (2010) also studied the dynamical evolution of escaped Plutinos in the GPC zone and found that they behave according to the four dynamical classes proposed by DB07. However, the resonance hopping mechanism and a high frequency of Kozai resonances in all the four classes are preferred. In particular, MMRs and Kozai resonances in the zone of $30<a<50$ au are more frequent than others. They also noted that some escaped Plutinos are captured again on the 3:2 MMR for some time before reaching their final state. The dynamical evolution of escaped Plutinos is very similar to that of SDOs 
in the GPC zone as can be seen by comparing the time-weighted distribution maps (see Fig. 3 in Di Sisto et al (2009)) with our Fig. 9 and 10

Horner and Lykawka (2010) evaluated the possibility of Neptune Trojans being a source of GPC. They found that $1 \mathrm{~km}$-sized Neptune Trojan enters the region of $q<30$ au every $\sim 60-200$ years, a rate well below the one from the SD of $3 \mathrm{~km}$-sized SDOs every 2 years. Volk (2013) calculated the supply rate of GPC and JFCs from each of the sub-populations in the TN region with numerical simulations, obtaining a fractional escape rate of $3 \times 10^{-11} \mathrm{yr}^{-1}$ for the $3: 2 \mathrm{MMR}$ and $10^{-10} \mathrm{yr}^{-1}$ for the $1: 2 \mathrm{MMR}$ and similar for the $5: 3$ and $7: 4$, well below the escape rate from the SD. Alexandersen et al (2016) estimate also populations of Neptunian and Uranian coorbitals, as well as objects in the $3: 1$ and $4: 1$ MMR with Neptune which are two orders of magnitude less than the Plutino population. Therefore, their contribution to the GPC and Centaur zones would be negligible.

However, with the recent surveys on the TN zone, several MMRs with Neptune have been discovered to be populated. Gladman et al (2012) discuss the MMRs in the TN zone using objects detected by CFEPS, and provide absolute population estimates for those resonances. They found that the 5:2 MMR could be as populated as the 3:2 whereas the 2:1 MMR has $\sim 4$ times less objects than the 3:2 and 5:2 MMRs. For the other MMR: 4:3, 5:3, 7:3, $5: 4,7: 4,3: 1$, and $5: 1$, they measure significant populations being the $5: 160 \%$ of plutinos. This last MMR was also found to be one of the most populous resonances by Pike et al (2015). By analyzing the resonant objects discovered by OSSOS survey, Volk et al (2016) found that the 2:1 MMR could be as populated as the 5:2 MMR and possibly as numerous as plutinos. Therefore, the real contribution of other MMRs than 3:2 could be as important as plutinos, but more work is needed in relation to their dynamical evolution to Centaurs and JFCs.

\subsection{From CTNOs}

Classical TNOs and Plutinos were the first to be discovered among TNOs, due to their relatively closeness and cold orbits. A few years after the first classical object was discovered in 1992 by David Jewitt (Jewitt et al 1992), Levison and Duncan (1997) performed the first dynamical evolution of CTNOs. They selected from a previous simulation, 20 particles that left the Kuiper Belt (KB) after being stable for over 1 Gyr. Thus, the orbits of these particles were representative of the orbits of objects currently leaving the classical TN region. They added clones of those particles and followed their dynamical evolution for 1 Gyr under the gravitational action of the Sun and the four Giant planets up to collision or ejection. They investigated the distribution and fates of objects which had left the KB in the current configuration of the Solar System. They found that the objects evolve inward from the KB being under the dynamical control of one planet at a time and keeping a very narrow range in the Tisserand parameter $(\mathrm{T})$ with respect to each control 
giant planet. Thus, they reach the Jupiter control with $\mathrm{T}$ just below 3 in a very narrow range as it is observed in JFCs. They also estimated a number of $\mathrm{km}$-sized ecliptic comets of $\sim 1.2 \times 10^{7}$.

Based on the study of the TN region as a source of JFCs and Centaurs, Volk (2013) obtained a fractional escape rate of $0.55 \times 10^{-10} \mathrm{~N} / \mathrm{yr}$ for the debiased CTNOs, a rate that is lower than our results for the escape rate from the SD. In fact, Volk (2013) found that the contribution to Centaurs from the $\mathrm{SD}$ is at least two times greater than that of CTNOs. Muñoz-Gutiérrez et al (2019) obtained a rate of injection from the CTNOs to the JFCs zone of $0.306 \times 10^{-10} \mathrm{~N} /$ year with the integration including 34 large TNOs and $0.261 \times$ $10^{-10} \mathrm{~N} /$ year without the large TNOs. Those rates are slightly smaller than the ones obtained by Volk (2013) since they calculated the escape rate and Muñoz-Gutiérrez et al (2019) calculated the injection to JFCs.

\subsection{From Jupiter Trojan asteroids}

Jupiter Trojans are asteroids trapped in the 1: 1 MMR with Jupiter and located around the $L_{4}$ and $L_{5}$ Lagrangian points on relatively stable orbits. However, they cover a space near $L_{4}$ and $L_{5}$ where zones of different scales of stability can be differentiated. This allows some Trojans to escape from the resonance on timescales that are shorter than the age of the Solar System (Levison et al 1997; Di Sisto et al 2014). Those escapees may contribute to populate other zones of the Solar System. The contribution of Jupiter Trojans to other Solar System populations was studied by Di Sisto et al (2019). They considered the observed Jupiter Trojan population and performed long-term numerical simulations to study the trajectories of those Trojans that leave the resonance. They obtained a constant escape rate from both Lagrangian points over time of $Y_{L_{4}}=7.0398 \times 10^{-11} \pm 8 \times 10^{-14} N_{T} / y r$ and $Y_{L_{5}}=7.5590 \times 10^{-11} \pm$ $13 \times 10^{-14} N_{T} / y r$, where $N_{T}$ is the cumulative number of Trojans. Di Sisto et al (2019) also found that $\sim 90 \%$ of escaped Trojans from $L_{4}$ and $L_{5}$ go through the GPC and Centaur zone and that the distribution of both spatial and angular orbital elements of escaped Trojans are similar to those of the observed Centaurs (see their Fig. 10). They obtained the number of Trojans ejected out of the resonance per year as a result of dynamical evolution considering the number of Trojans and their size distribution and the member number asymmetry between the $L_{4}$ and $L_{5}$ swarms. Additionally, including the rate of escape from the resonance by collisional evolution from de Elía and Brunini (2007), they calculated the number of escaped Trojans in each minor body population and found that the contribution of escaped Trojans in the Centaur zone would be minor.

The contribution from both Jupiter Trojan swarms to GPC and Centaurs are plotted together with Plutinos and SDOs in Fig. 14 and 15. 


\subsection{Retrograde GPC and Centaurs}

Other sources of Centaurs apart from TNOs have been proposed and studied, especially in order to explain particular cases. There is a very low probability for a high inclination Centaur or even a retrograde one to have its origin in the TN region (Volk and Malhotra 2013). Brasser et al (2012) showed that Centaurs with inclinations $i>70^{\circ}$ and $q>15 \mathrm{au}$, originate mainly in the Oort Cloud being decoupled from it by the gravitational perturbations of Uranus and Neptune alone. Those authors estimated that there are between 1 and 200 Centaurs with absolute magnitude $H<8\left(D \gtrsim 150 \mathrm{~km}\right.$ for $\left.p_{v}=0.05\right)$ in that range of $i$ and $q$. The case of retrograde Centaurs was analyzed by de la Fuente Marcos and de la Fuente Marcos (2014) who concluded that they formed an heterogeneous group that may have its origin in the Oort cloud but that other sources or dynamical mechanisms that enlarge the inclinations can not be ruled out. Those Centaurs are trapped in transient retrograde, mostly higher-order resonances with the giant planets, leading to chaotic diffusion and thus making their orbits dynamically unstable. This is also observed for the retrograde GPC in our dynamical evolution of observed objects in Sect. 2.2 Nesvorný et al (2017) performed numerical simulations of the early evolution of the Solar System in which cometary reservoirs were formed and evolved, and found that the SD (with a flat inclination distribution) is the source of ecliptic comets, while the Oort cloud is the source of Halley-type comets (HTCs) with an isotropic inclination distribution. Nesvorny et al (2019) performed a study directed to test a model of the early evolution of the outer Solar System by running it through the OSSOS survey simulator. They started their simulation with an original flat planetesimal disk below $30 \mathrm{au}$, and followed its evolution from the onset of Neptune's migration to the present time. They obtained that in the last Gyr, 11\% of Centaurs evolved from the region with $a>5000$ au. However, only some very-high-inclination Centaurs can be explained by their simulation and then other sources of very-high-inclination and retrograde Centaurs are needed.

\subsection{Region interior to Saturn and Jupiter crossers}

The region between Jupiter and Saturn has been investigated by a number of authors, analyzing both the physical and dynamical processes of its objects. However, the Jupiter crossers zone is difficult to address completely. The numerical simulations that study the TNOs as a source of GPC or Centaurs generally consider only the perturbations of the Giant planets; they discard the particles when they reach Jupiter's orbit since the terrestrial planets and a lower integration step is necessary for accurate results on dynamical evolution. On the other hand, a purely dynamical simulation would be no longer appropriate in the region interior to Jupiter's orbit (the JFC zone) because of erosion processes of icy objects at this distance. Therefore, a general self-consistent physico-dynamical model from the $\mathrm{TN}$ zone to the JFC zone is difficult to 
address. This problem was approached by Di Sisto et al (2009) who continued the integration of the particles that arrived at Jupiter in the simulation of DB07. In this work, Di Sisto et al (2009) integrated those particles plus clones under the perturbation of all the planets and by considering also non gravitational forces and a model for sublimation and splitting of comets. They focused their study in JFCs $(P<20$ yrs and $2<T<3.1)$ by obtaining their distribution in the orbital element space and also their expected number in regions of perihelion distances. They obtained a mean lifetime of JFCs with $D>2 \mathrm{~km}$ and $q<1.5$ au of about 150200 revolutions $(\sim 1000 \mathrm{yrs})$, for $q<2.5 \mathrm{au} \sim 300-450$ revolutions and a total population of JFCs with $D>2$ $\mathrm{km}$ within Jupiter's zone of $450 \pm 50$. However, they found a greater population of non-JFCs (those that don't fulfill the conditions $T>2$ and $P<20$ yr simultaneously), which would be 4 times greater for Jupiter-crossing orbits of comets of $D>2 \mathrm{~km}$. Thus leading to a whole population of JFCs + non-JFCs of $D>2 \mathrm{~km}$ of $2250 \pm 250$ in the zone of $q<5.2$. Nesvorný et al (2017) performed numerical simulations of a primordial Solar System, in which cometary reservoirs are formed and evolved over 4.5 Gyr. From this simulation they found the current population of JFCs and also HTCs. By accounting for the physical lifetime of active comets through different parametrizations, and comparing with observations, they inferred a mean lifetime of JFCs with $q<2.5$ au of $\sim 300-800$ revolutions, consistent with Di Sisto et al (2009). They also found that the number of JFCs with $D>10 \mathrm{~km}$ is $\sim 1-2$.

Di Sisto et al (2009) also found that larger comets usually return to the Centaur zone in their evolution, but smaller ones ( $1-\mathrm{km}$ comets), suffer a quick erosion reaching a minimum proposed radius (100 $\mathrm{mts})$ before they can evolve into other dynamical states. Therefore, the Centaur zone near Jupiter is partially re-filled by comets and this is strongly dependent on the size. Working with the numerical simulations of Di Sisto et al (2009), we found that objects that return to the Centaur zone from the JFC zone experience a very quick dynamical evolution among the Giant Planets until ejection. Their dynamical mean lifetime in the region of $q>5.2$ au and $a<30$ au is of $0.4 \mathrm{Myr}$, very similar to that of escaped Jupiter Trojans. Also their dynamical evolution is similar to that of JTs (see Fig. 3 in Di Sisto et al (2019)).

Brasser and Wang (2015) performed numerical simulations of the evolution of SDOs until they became visible JFCs, keeping track of their number of perihelion passages with $q<2.5$ au. They used a simple fading law applied to JFCs that depended on the number of revolutions. From their simulation and observational data on JFCs, they estimate that the steady-state number of active JFCs with $q<2.5$ au and diameter $D>2.3 \mathrm{~km}$ is $294_{-235}^{+556}$. This is a larger value than estimates by Levison et al (1997) and Di Sisto et al (2009), but given the large uncertainty, it can be considered to be in the same range as the latter.

On the other hand, in the Jupiter-Saturn region there are also some signs of sublimation in some objects in contrast to others that remain inactive. Fernández et al (2018) addressed a sub-population of Centaurs in the JupiterSaturn region, with the aim of investigating the dynamical evolution and end 
states of active and inactive Centaurs. The authors performed the study with numerical simulations integrating two samples of real Centaurs (one for active Centaurs and the other for inactive ones) and clones under the gravitational action of the Sun and the planets from Venus to Neptune. They concluded that inactive and active Centaurs have different dynamical behaviors and different median dynamical lifetimes that could explain the presence of activity in some Centaurs and the lack of it in others. In addition, they show that probably evolution (such as a recent drastic drop in perihelion distance) and not intrinsic physical differences explains the display or not of activity of Centaurs in the Jupiter-Saturn region.

Close encounters with Jupiter or Saturn could turn out to be drastic for a comet-like object. In a close encounter of a comet with one of these giant planets, tidal effects can become so important that they can cause the fragmentation of the body and its eventual collision with the planet, as was the case of Shoemaker Levy 9 with Jupiter in 1992-1994. Also, they could be important on the preservation of the rings around Centaurs. In 2014, Braga-Ribas et al (2014) discovered around Chariklo, the first ring around a Centaur. It was an unexpected discovery, since given the chaotic dynamical evolution of Centaurs, with close encounters with the giant planets, it should be difficult to retain a ring. Of course this depends on the competition between the formation processes (probably by collisions) and destruction processes. Numerical studies by Araujo et al (2016) and Wood et al (2017) evaluated the encounters of Chariklo (and clones) within 1 Hill radius of the planet and found that they have little effect on the rings and that they could survive through its evolution as a Centaur. However, Wood et al (2018) found that a close encounter has a noticeable effect on the ring of a small body if it occurs at a distance within $\approx 1.8$ tidal disruption distances (the distance where tidal forces can disrupt a small bodyring particle), which is much smaller that the Hill radius. Therefore, the presence and survival of rings on Centaurs is strongly dependent on their dynamical evolution in the giant planet zone.

Therefore, the region interior to Saturn up to Jupiter, is a complex zone to evaluate the dynamical behavior of Centaurs. Physical models are needed together with dynamical models, or at least it is necessary to consider the possible shortening of the physical lifetimes of small objects by the possible presence of activity. However, the dynamical evolution is fast. In the region of low-eccentricity orbits with $q>5.4$ au and $Q<7.8$ au, Sarid et al (2019) found a niche which they called a Gateway for transition between Centaurs and JFCs. They found that $77 \%$ of objects in this Gateway region will become or have already been JFCs, and nearly half of all JFCs will pass through this Gateway region before experiencing significant sublimation. Therefore, this is an important discovery in relation to the dynamical link between populations, but also for the purposes of the conditions to develop comet activity. 


\section{General model from all GPC and Centaur sources}

From the results obtained in the previous sections, we show the combined contribution of SDOs, Plutinos and Jupiter Trojans to GPC and Centaurs. In Figs. 14 and 15 the cumulative number of GPC and Centaurs respectively are plotted against diameter. As can be seen from those figures, the GPC



Fig. 14 Cumulative number of Giant Planet Crossers $\left(N_{G P C}(>D)\right)$ vs diameter $D$ from SDOs (red curves), Plutinos (green curves) and Jupiter Trojans (blue curves). The gray dots correspond to the observed population. For the observed population we use an albedo $p_{v}=0.06$ to convert absolute magnitude to diameter.

and Centaur zones are mainly fed by SDOs from the TN zone, and the other contributions are secondary. It is notable that both the intrinsic number of GPC and Centaurs proposed by our model are respectively greater than the real observed numbers. However, the difference between model and observation for Centaurs is smaller than for GPCs. This is due to biases in the discovered populations which tend to favor the detection of Centaurs over GPCs, since the latter are more distant objects. Hence, there could be a great number of GPCs to be discovered yet.

Those results are very sensitive to the size distribution of the source populations, and this is not a concluded topic. Especially for small diameters, where the SFD of objects may have a break, the results are very variable. We have shown in Fig. 14 and 15 limit values for the power law index of the size distributions at smaller sizes. This uncertainty leads to, for example, that for $D<5 \mathrm{~km}$, depending on the size distribution index, the contribution from 


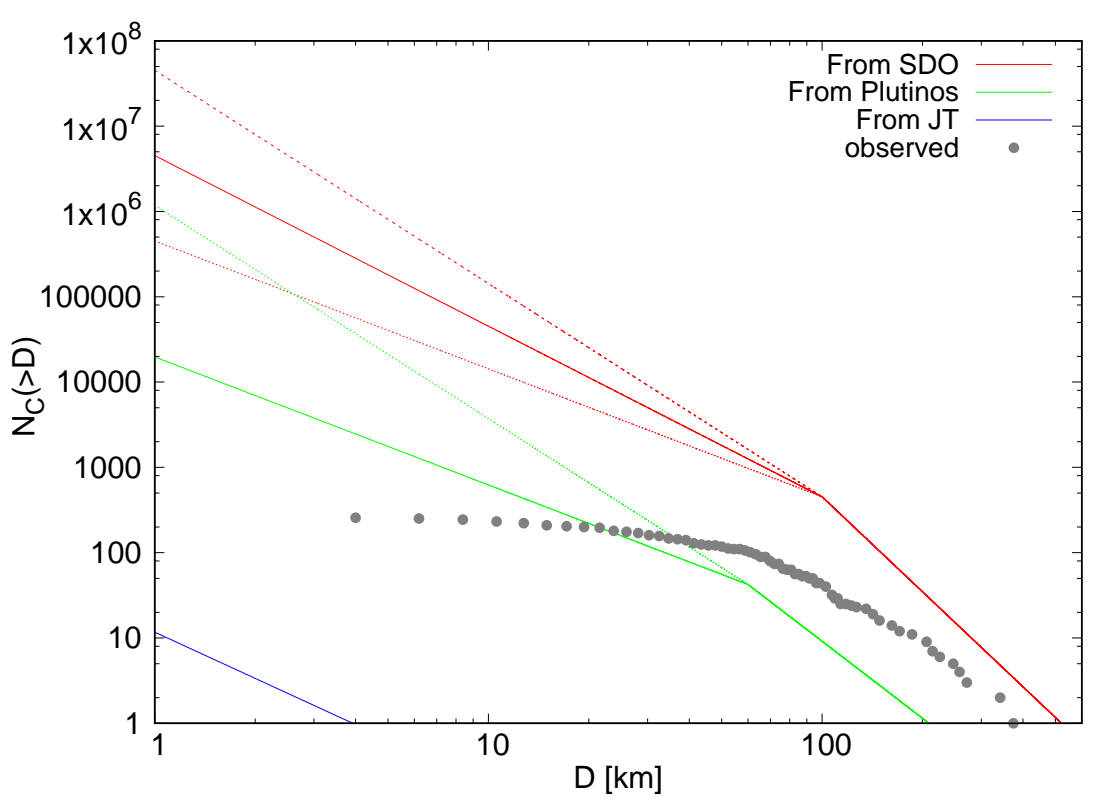

Fig. 15 Cumulative number of Centaurs $\left(N_{C}(>D)\right)$ vs diameter $D$ from SDOs (red curves), Plutinos (green curves) and Jupiter Trojans (blue curves). The gray dots correspond to the observed population. For the observed population we use an albedo $p_{v}=0.06$ to convert absolute magnitude to diameter.

Plutinos could be greater than the contribution from SDOs. Although the SDOs and Plutinos are two dynamically excited populations and could probably have the same initial index in their size distribution (or at least similar), the collisional evolution of Plutinos could have changed this. Moreover, for very small objects $(\lesssim 1 \mathrm{~km})$, there may be an additional break in the SFD slopes of TNOs as was suggested from cratering counts (Di Sisto and Zanardi 2013; Singer et al 2019), but more studies are needed for those small TNOs. Therefore, this is an open scenario, and more observations and studies are needed to assess completely the size distribution at small diameters.

On the one hand, secondary sources may explain some peculiarities, probably related in particular to different Centaur compositions. Although JT are a minor source of GPC and Centaurs, Di Sisto et al (2019) found that some NEO-JFC orbits, Encke-type comets and impacts on Jupiter as the Shoemaker Levy 9 case can be explained by the dynamical evolution of escaped Trojans. On the other hand, in their path to being ejected from the Solar System, JT and JFCs go faster through the giant planet zone than in their way inward from the SD and other secondary sources. They are also affected by the physical processes they suffered when they were within the orbit of Jupiter, so they could give rise to different types of Centaurs mainly in the area near Jupiter and Saturn. 


\section{Conclusions}

For the present review we have performed new numerical simulations and calculations of the Giant Planetary Crossers studying their number and their evolution from their sources, considering the current configuration of the Solar System.

From the dynamical evolution of SDOs, we have calculated the number of Centaurs predicted by our model. In comparison, the recent estimates by OSSOS team are somewhat lower than ours for the diameters studied (see Fig. 13).

From the results obtained in the numerical simulation of SDOs and from previous works, we obtain the contribution of SDOs, Plutinos and Jupiter Trojans (JT) to GPC and Centaurs as shown in Figs. 14 and 15. From those Figs., the SD is the main source of prograde GPC and Centaurs as was first suggested by Duncan and Levison (1997) and found also by other subsequent studies as DB07 and Volk (2013). For example, there are 9600 GPCs from the SD with $D>100 \mathrm{~km}$ and $10^{8}$ with $D>1 \mathrm{~km}$ (with a differential SFD index of 3$)$.

The cumulative number of escaped Plutinos in the GPC zone lies between one and two orders of magnitude less than that from the SD. However, those results are very sensitive to the size distribution of the source populations and especially for small diameters, where the SFD of objects may have a break. The uncertainty at small sizes could lead to an important contribution from Plutinos if the index of the SFD of Plutinos was greater than that of the SDOs. New observations and works on other MMRs in the TN zone Gladman et al 2012; Pike et al 2015; Volk et al 2016) indicate that some MMR, specially the $2: 1$ and 5:2, could be as populated as plutinos, and therefore they have to be in consideration as possible contributors to Centaurs and JFCs. The contribution from JT can be considered negligible although it may explain some peculiarities.

The observed number of GPCs and Centaurs are well below our model due to observational biases in the surveys. Therefore, there could be many GPCs and Centaurs to be discovered yet.

The dynamical evolution of TNOs in general through the GPC zone and then to the JFC region, can be seen as a hand off from the gravitational control of one planet to another as was first shown by Levison and Duncan (1997). The mean lifetime of SDOs in the GPC zone is of 68 Myr. There is a correlation between the particles lifetime in the GPC zone and their perihelion distance, where the greater the perihelion, the longer the mean lifetime. This is connected with a slower evolution of objects at larger perihelion, where the variation of $q$ is small in the region between the orbits of Saturn and Neptune. We detect transfers between mean motion resonances, known as resonance sticking, in the zone of $\sim 30$ au $<a \lesssim 200$ au. Beyond 200 au, the general dynamical evolution is a low increase of semimajor axis but keeping the perihelion distances near Neptune. Thus, the objects are continuously entering 
and leaving the GPC zone up to ejection (injection to the GPC zone of those particles is more rare).

The initial inclination affects the mean lifetime of particles as GPC, being the ones with high initial inclinations the ones that survived longer time in the GPC zone. In the Jupiter-Saturn region, the dynamical evolution is faster. Different dynamical behaviors were also found by Fernández et al (2018) in inactive and active Centaurs, that are also connected with different median dynamical lifetimes and that may help to explain the activity or lack of activity in some Centaurs.

From our simulations, the initial particles (i.e. objects in the SD) which had initial inclinations lower than $55^{\circ}$, evolved into prograde GPC and Centaurs. Thus, with our model we were not able to generate any prograde GPC with inclinations greater than $60^{\circ}$ nor a retrograde GPC. Therefore, as was found by other authors, retrograde Centaurs probably did not come from the SD but from other source such as the Oort Cloud (e.g. Brasser et al (2012), Volk and Malhotra (2013)). In fact, we have performed numerical simulations of the dynamical evolution of observed GPC, within which there are 17 retrograde objects that show different properties than the prograde population. In this study, the observed prograde population has a similar behavior to those GPCs that enter from the SD (from our model), but retrograde GPCs have a comparatively shorter median lifetime. Thus, retrograde GPCs experience a faster evolution than prograde ones. However, it is probable that this faster evolution is due to the fact that the majority of retrograde GPCs (15 of the 17) have low perihelion values and then, lower lifetimes. In fact the two retrograde objects with high perihelion distances have long lifetimes, greater than 600 Myr. We also note that some retrograde objects experience MMR captures as well.

\section{Acknowledgments}

We acknowledge the financial support given by IALP, CONICET and of Agencia de Promocin Científica and also the FCAGLP for extensive use of their computing facilities. We would like to thank two anonymous reviewers for their detailed comments and suggestions which helped us to significantly improve this article, and also Tabaré Gallardo for valuable discussion about encounter conditions and Opik encounter probability.

\section{References}

Adams ER, Gulbis AAS, Elliot JL, Benecchi SD, Buie MW, Trilling DE, Wasserman LH (2014) De-biased Populations of Kuiper Belt Objects from the Deep Ecliptic Survey. Astronomical Journal 148(3):55, DOI 10.1088/ 0004-6256/148/3/55, 1311.3250 
Alexandersen M, Gladman B, Greenstreet S, Kavelaars JJ, Petit JM, Gwyn S (2013) A Uranian Trojan and the Frequency of Temporary Giant-Planet Co-Orbitals. Science 341(6149):994-997, DOI 10.1126/science. 1238072, 1303.5774

Alexandersen M, Gladman B, Kavelaars JJ, Petit JM, Gwyn SDJ, Shankman CJ, Pike RE (2016) A Carefully Characterized and Tracked TransNeptunian Survey: The Size distribution of the Plutinos and the Number of Neptunian Trojans. Astronomical Journal 152(5):111, DOI 10.3847/ 0004-6256/152/5/111, 1411.7953

Araujo RAN, Sfair R, Winter OC (2016) The Rings of Chariklo under Close Encounters with the Giant Planets. The Astrophysical Journal 824(2):80, DOI 10.3847/0004-637X/824/2/80,1604.07323

Bailey BL, Malhotra R (2009) Two dynamical classes of Centaurs. Icarus 203(1):155-163, DOI 10.1016/j.icarus.2009.03.044,0906.4795

Bannister MT, Gladman BJ, Kavelaars JJ, Petit JM, Volk K, Chen YT, Alexandersen M, Gwyn SDJ, Schwamb ME, Ashton E, Benecchi SD, Cabral N, Dawson RI, Delsanti A, Fraser WC, Granvik M, Greenstreet S, GuilbertLepoutre A, Ip WH, Jakubik M, Jones RL, Kaib NA, Lacerda P, Van Laerhoven C, Lawler S, Lehner MJ, Lin HW, Lykawka PS, Marsset M, Murray-Clay R, Pike RE, Rousselot P, Shankman C, Thirouin A, Vernazza P, Wang SY (2018) OSSOS. VII. 800+ Trans-Neptunian Objects - The Complete Data Release. The Astrophysical Journal Supplement Series 236:18, 1805.11740

Bernstein GM, Trilling DE, Allen RL, Brown ME, Holman M, Malhotra R (2004) The Size Distribution of Trans-Neptunian Bodies. Astronomical Journal 128:1364-1390, astro-ph/0308467

Bierhaus EB, Dones L (2015) Craters and ejecta on Pluto and Charon: Anticipated results from the New Horizons flyby. Icarus 246:165-182, DOI 10.1016/j.icarus.2014.05.044

Braga-Ribas F, Sicardy B, Ortiz JL, Snodgrass C, Roques F, Vieira-Martins R, Camargo JIB, Assafin M, Duffard R, Jehin E, Pollock J, Leiva R, Emilio M, Machado DI, Colazo C, Lellouch E, Skottfelt J, Gillon M, Ligier N, Maquet L, Benedetti-Rossi G, Gomes AR, Kervella P, Monteiro H, Sfair R, El Moutamid M, Tancredi G, Spagnotto J, Maury A, Morales N, Gil-Hutton R, Roland S, Ceretta A, Gu SH, Wang XB, Harpsøe K, Rabus M, Manfroid J, Opitom C, Vanzi L, Mehret L, Lorenzini L, Schneiter EM, Melia R, Lecacheux J, Colas F, Vachier F, Widemann T, Almenares L, Sand ness RG, Char F, Perez V, Lemos P, Martinez N, Jørgensen UG, Dominik M, Roig F, Reichart DE, Lacluyze AP, Haislip JB, Ivarsen KM, Moore JP, Frank NR, Lambas DG (2014) A ring system detected around the Centaur (10199) Chariklo. Nature 508(7494):72-75, DOI 10.1038/nature13155, 1409.7259

Brasser R, Wang JH (2015) An updated estimate of the number of Jupiterfamily comets using a simple fading law. Astronomy and Astrophysics 573:A102, DOI 10.1051/0004-6361/201423687, 1412.1198

Brasser R, Schwamb ME, Lykawka PS, Gomes RS (2012) An Oort cloud origin for the high-inclination, high-perihelion Centaurs. Monthly Notices of the 
Royal Astronomical Society 420(4):3396-3402, DOI 10.1111/j.1365-2966. 2011.20264.x, 1111.7037

Brown ME (2001) The Inclination Distribution of the Kuiper Belt. The Astronomical Journal 121(5):2804-2814, DOI 10.1086/320391

Chen YT, Lin HW, Holman MJ, Payne MJ, Fraser WC, Lacerda P, Ip WH, Chen WP, Kudritzki RP, Jedicke R, Wainscoat RJ, Tonry JL, Magnier EA, Waters C, Kaiser N, Wang SY, Lehner M (2016) Discovery of a New Retrograde Trans-Neptunian Object: Hint of a Common Orbital Plane for Low Semimajor Axis, High-inclination TNOs and Centaurs. The Astrophysical Journal Letters 827(2):L24, DOI 10.3847/2041-8205/827/2/L24, 1608.01808

de Elía GC, Brunini A (2007) Collisional and dynamical evolution of the $\mathrm{L}_{4}$ Trojan asteroids. Astronomy and Astrophysics 475(1):375-389

de Elía GC, Brunini A, di Sisto RP (2008) Collisional and dynamical evolution of Plutinos. Astronomy and Astrophysics 490(2):835-842, DOI 10.1051/0004-6361:200809865

de la Fuente Marcos C, de la Fuente Marcos R (2014) Large retrograde Centaurs: visitors from the Oort cloud? Astrophysics and Space Science 352(2):409-419, 1406.1450

Di Sisto RP, Brunini A (2007) The origin and distribution of the Centaur population. Icarus 190:224-235

Di Sisto RP, Brunini A (2011) Origin of craters on Phoebe: comparison with Cassini's data. Astronomy \& Astrophysics 534:A68, 1108.3808

Di Sisto RP, Zanardi M (2013) The production of craters on the mid-sized Saturnian satellites by Centaur objects. Astronomy \& Astrophysics 553:A79

Di Sisto RP, Fernández JA, Brunini A (2009) On the population, physical decay and orbital distribution of Jupiter family comets: Numerical simulations. Icarus 203(1):140-154

Di Sisto RP, Brunini A, de Elía GC (2010) Dynamical evolution of escaped plutinos, another source of Centaurs. Astronomy \& Astrophysics 519:A112, 1005.3267

Di Sisto RP, Ramos XS, Beaugé C (2014) Giga-year evolution of Jupiter Trojans and the asymmetry problem. Icarus 243:287-295, 1604.05331

Di Sisto RP, Ramos XS, Gallardo T (2019) The dynamical evolution of escaped Jupiter Trojan asteroids, link to other minor body populations. Icarus $319: 828-839,1811.00352$

Dones L, Brasser R, Kaib N, Rickman H (2015) Origin and Evolution of the Cometary Reservoirs. Space Science Reviews 197(1-4):191-269, DOI 10.1007/s11214-015-0223-2

Duncan M, Quinn T, Tremaine S (1988) The origin of short-period comets. The Astrophysical Journal Letters 328:L69-L73

Duncan MJ, Levison HF (1997) A scattered comet disk and the origin of Jupiter family comets. Science 276:1670-1672

Edgeworth KE (1938) The evolution of the solar system. Unpublished manuscript, Trustees of the National Library of Ireland, Dublin Manuscript Nos $16869 / 47$ and /48 
Elliot JL, Kern SD, Clancy KB, Gulbis AAS, Millis RL, Buie MW, Wasserman LH, Chiang EI, Jordan AB, Trilling DE, Meech KJ (2005) The Deep Ecliptic Survey: A Search for Kuiper Belt Objects and Centaurs. II. Dynamical Classification, the Kuiper Belt Plane, and the Core Population. Astronomical Journal 129:1117-1162

Fernández JA (1980) On the existence of a comet belt beyond Neptune. Monthly Notices of the Royal Astronomical Society 192:481-491

Fernandez JA, Ip WH (1984) Some dynamical aspects of the accretion of Uranus and Neptune: The exchange of orbital angular momentum with planetesimals. Icarus 58(1):109-120

Fernández JA, Gallardo T, Brunini A (2002) Are There Many Inactive JupiterFamily Comets among the Near-Earth Asteroid Population? Icarus 159:358 368

Fernández JA, Gallardo T, Brunini A (2004) The scattered disk population as a source of Oort cloud comets: evaluation of its current and past role in populating the Oort cloud. Icarus 172:372-381

Fernández JA, Helal M, Gallardo T (2018) Dynamical evolution and end states of active and inactive Centaurs. Planetary and Space Science 158:6-15, 1805.05994

Fraser WC, Kavelaars JJ (2009) The Size Distribution of Kuiper Belt Objects for $\mathrm{D} \gtrsim 10 \mathrm{~km}$. Astronomical Journal 137:72-82,0810.2296

Fuentes CI, Holman MJ (2008) a SUBARU Archival Search for Faint TransNeptunian Objects. Astronomical Journal 136:83-97

Fuentes CI, George MR, Holman MJ (2009) A Subaru Pencil-Beam Search for $\mathrm{m}_{R} \sim 27$ Trans-Neptunian Bodies. Astrophysical Journal 696:91-95, 0809.4166

Gil-Hutton R, Licandro J, Pinilla-Alonso N, Brunetto R (2009) The transNeptunian object size distribution at small sizes. Astronomy and Astrophysics 500:909-916

Gladman B, Kavelaars J, Petit JM, Ashby MLN, Parker J, Coffey J, Jones RL, Rousselot P, Mousis O (2009) Discovery of the First Retrograde Transneptunian Object. The Astrophysical Journal Letters 697(2):L91-L94, DOI 10.1088/0004-637X/697/2/L91

Gladman B, Lawler SM, Petit JM, Kavelaars J, Jones RL, Parker JW, Van Laerhoven C, Nicholson P, Rousselot P, Bieryla A, Ashby MLN (2012) The Resonant Trans-Neptunian Populations. The Astronomical Journal 144(1):23, DOI 10.1088/0004-6256/144/1/23, 1205.7065

Gulbis AAS, Elliot JL, Adams ER, Benecchi SD, Buie MW, Trilling DE, Wasserman LH (2010) Unbiased Inclination Distributions for Objects in the Kuiper Belt. The Astronomical Journal 140(2):350-369, DOI 10.1088/ 0004-6256/140/2/350, 1005.1719

Horner J, Lykawka PS (2010) The Neptune Trojans - a new source for the Centaurs? Monthly Notices of the Royal Astronomical Society 402:13-20, 1002.2022

Jewitt D, Luu J, Marsden BG (1992) 1992 QB1. IAU CircNo 5622 5611:1

Kirchoff MR, Schenk P (2010) Impact cratering records of the mid-sized, icy 
saturnian satellites. Icarus 206(2):485-497, DOI 10.1016/j.icarus.2009.12. 007

Kowal CT, Gehrels T (1977) Slow-Moving Object Kowal. IAU Circ 3129:1

Kuiper GP (1951) On the Origin of the Solar System. Proceedings of the National Academy of Science 37:1-14

Lawler SM, Kavelaars JJ, Alexandersen M, Bannister MT, Gladman B, Petit JM, Shankman C (2018a) OSSOS: X. How to use a Survey Simulator: Statistical Testing of Dynamical Models Against the Real Kuiper Belt. Frontiers in Astronomy and Space Sciences 5:14, DOI 10.3389/fspas.2018.00014, 1802.00460

Lawler SM, Shankman C, Kavelaars JJ, Alexand ersen M, Bannister MT, Chen YT, Gladman B, Fraser WC, Gwyn S, Kaib N, Petit JM, Volk K (2018b) OSSOS. VIII. The Transition between Two Size Distribution Slopes in the Scattering Disk. Astronomical Journal 155(5):197, DOI 10.3847/1538-3881/ aab8ff, 1803.07521

Levison HF, Duncan MJ (1997) From the Kuiper Belt to Jupiter-Family Comets: The Spatial Distribution of Ecliptic Comets. Icarus 127(1):13-32, DOI 10.1006/icar.1996.5637

Levison HF, Shoemaker EM, Shoemaker CS (1997) Dynamical evolution of Jupiter's Trojan asteroids. Nature 385:42-44

Lykawka PS, Mukai T (2007) Resonance sticking in the scattered disk. Icarus 192(1):238-247, DOI 10.1016/j.icarus.2007.06.007,0707.4301

Malhotra R (1993) The origin of Pluto's peculiar orbit. Nature 365(6449):819 821

Malhotra R (1995) The Origin of Pluto's Orbit: Implications for the Solar System Beyond Neptune. Astronomical Journal 110:420, DOI 10.1086/117532, astro-ph/9504036

Meech KJ, Belton MJS (1990) The Atmosphere of 2060 Chiron. Astronomical Journal 100:1323, DOI 10.1086/115600

Moore JM, McKinnon WB, Spencer JR, Howard AD, Schenk PM, Beyer RA, Nimmo F, Singer KN, Umurhan OM, White OL, Stern SA, Ennico K, Olkin CB, Weaver HA, Young LA, Binzel RP, Buie MW, Buratti BJ, Cheng AF, Cruikshank DP, Grundy WM, Linscott IR, Reitsema HJ, Reuter DC, Showalter MR, Bray VJ, Chavez CL, Howett CJA, Lauer TR, Lisse CM, Parker AH, Porter SB, Robbins SJ, Runyon K, Stryk T, Throop HB, Tsang CCC, Verbiscer AJ, Zangari AaM, Chaikin AL, Wilhelms DE, Bagenal F, Gladstone GR, Andert T, Andrews J, Banks M, Bauer B, Bauman J, Barnouin OS, Bedini P, Beisser K, Bhaskaran S, Birath E, Bird M, Bogan DJ, Bowman A, Brozovic M, Bryan C, Buckley MR, Bushman SS, Calloway A, Carcich B, Conard S, Conrad CA, Cook JC, Custodio OS, Ore CMD, Deboy C, Dischner ZJB, Dumont P, Earle AM, Elliott HA, Ercol J, Ernst CM, Finley T, Flanigan SH, Fountain G, Freeze MJ, Greathouse T, Green JL, Guo Y, Hahn M, Hamilton DP, Hamilton SA, Hanley J, Harch A, Hart HM, Hersman CB, Hill A, Hill ME, Hinson DP, Holdridge ME, Horanyi M, Jackman C, Jacobson RA, Jennings DE, Kammer JA, Kang HK, Kaufmann DE, Kollmann P, Krimigis SM, Kusnierkiewicz D, Lee JE, Lindstrom KL, 
Lunsford AW, Mallder VA, Martin N, McComas DJ, McNutt RL, Mehoke D, Mehoke T, Melin ED, Mutchler M, Nelson D, Nunez JI, Ocampo A, Owen WM, Paetzold M, Page B, Parker JW, Pelletier F, Peterson J, Pinkine N, Piquette M, Protopapa S, Redfern J, Roberts JH, Rogers G, Rose D, Retherford KD, Ryschkewitsch MG, Schindhelm E, Sepan B, Soluri M, Stanbridge D, Steffl AJ, Strobel DF, Summers ME, Szalay JR, Tapley M, Taylor A, Taylor H, Tyler GL, Versteeg MH, Vincent M, Webbert R, Weidner S, Weigle GE, Whittenburg K, Williams BG, Williams K, Williams S, Woods WW, Zirnstein E (2016) The geology of Pluto and Charon through the eyes of New Horizons. Science 351(6279):1284-1293, DOI 10.1126/science.aad7055, 1604.05702

Morbidelli A (1997) Chaotic Diffusion and the Origin of Comets from the 2/3 Resonance in the Kuiper Belt. Icarus 127:1-12

Morbidelli A, Emel'yanenko VV, Levison HF (2004) Origin and orbital distribution of the trans-Neptunian scattered disc. Monthly Notices of the Royal Astronomical Society 355(3):935-940, DOI 10.1111/j.1365-2966.2004.08372. $\mathrm{x}$

Muñoz-Gutiérrez MA, Peimbert A, Pichardo B, Lehner MJ, Wang SY (2019) The Contribution of Dwarf Planets to the Origin of Jupiter Family Comets. The Astronomical Journal 158(5):184, DOI 10.3847/1538-3881/ab4399, 1909.04861

Nesvorný D (2015) Jumping Neptune Can Explain the Kuiper Belt Kernel. Astronomical Journal 150(3):68, DOI 10.1088/0004-6256/150/3/68, 1506.06019

Nesvorný D, Morbidelli A (2012) Statistical Study of the Early Solar System's Instability with Four, Five, and Six Giant Planets. The Astronomical Journal 144(4):117, 1208.2957

Nesvorný D, Roig F, Ferraz-Mello S (2000) Close Approaches of TransNeptunian Objects to Pluto Have Left Observable Signatures on Their Orbital Distribution. Astronomical Journal 119(2):953-969, DOI 10.1086/ 301208

Nesvorný D, Vokrouhlický D, Dones L, Levison HF, Kaib N, Morbidelli A (2017) Origin and Evolution of Short-period Comets. The Astrophysical Journal 845(1):27, DOI 10.3847/1538-4357/aa7cf6, 1706.07447

Nesvorny D, Vokrouhlicky D, Stern AS, Davidsson B, Bannister MT, Volk K, Chen YT, Gladman BJ, Kavelaars JJ, Petit JM, Gwyn SDJ, Alexandersen M (2019) OSSOS IXX: Testing Early Solar System Dynamical Models using OSSOS Centaur Detections. arXiv e-prints arXiv:1907.10723,1907.10723

Parker AH, Kavelaars JJ (2010a) Pencil-beam surveys for trans-neptunian objects: Limits on distant populations. Icarus 209:766-770, 1004.3288

Parker AH, Kavelaars JJ (2010b) Pencil-Beam Surveys for Trans-Neptunian Objects: Novel Methods for Optimization and Characterization. Publications of the Astronomical Society of the Pacific 122:549, 1004.3287

Petit JM, Kavelaars JJ, Gladman BJ, Jones RL, Parker JW, Bieryla A, Van Laerhoven C, Pike RE, Nicholson P, Ashby MLN, Lawler SM (2017) The Canada-France Ecliptic Plane Survey (CFEPS) - High-latitude Component. 
The Astronomical Journal 153(5):236, DOI 10.3847/1538-3881/aa6aa5, 1608.02873

Pike RE, Kavelaars JJ, Petit JM, Gladman BJ, Alexandersen M, Volk K, Shankman CJ (2015) The 5:1 Neptune Resonance as Probed by CFEPS: Dynamics and Population. The Astronomical Journal 149(6):202, DOI 10. 1088/0004-6256/149/6/202,1504.08041

Robbins SJ, Runyon K, Singer KN, Bray VJ, Beyer RA, Schenk P, McKinnon WB, Grundy WM, Nimmo F, Moore JM, Spencer JR, White OL, Binzel RP, Buie MW, Buratti BJ, Cheng AF, Linscott IR, Reitsema HJ, Reuter DC, Showalter MR, Tyler GL, Young LA, Olkin CB, Ennico KS, Weaver HA, Stern SA (2018) Investigation of Charon's Craters With Abrupt Terminus Ejecta, Comparisons With Other Icy Bodies, and Formation Implications. Journal of Geophysical Research (Planets) 123(1):20-36, DOI 10.1002/2017JE005287

Sarid G, Volk K, Steckloff JK, Harris W, Womack M, Woodney LM (2019) 29P/Schwassmann-Wachmann 1, A Centaur in the Gateway to the Jupiterfamily Comets. The Astrophysical Journal Letters 883(1):L25, DOI 10.3847/ 2041-8213/ab3fb3, 1908.04185

Shankman C, Kavelaars J, Gladman BJ, Alexand ersen M, Kaib N, Petit JM, Bannister MT, Chen YT, Gwyn S, Jakubik M, Volk K (2016) OSSOS. II. A Sharp Transition in the Absolute Magnitude Distribution of the Kuiper Belt's Scattering Population. Astronomical Journal 151(2):31, DOI 10.3847/ 0004-6256/151/2/31, 1511.02896

Singer KN, McKinnon WB, Gladman B, Greenstreet S, Bierhaus EB, Stern SA, Parker AH, Robbins SJ, Schenk PM, Grundy WM, Bray VJ, Beyer RA, Binzel RP, Weaver HA, Young LA, Spencer JR, Kavelaars JJ, Moore JM, Zangari AM, Olkin CB, Lauer TR, Lisse CM, Ennico K, New Horizons Geology GaISTT, New Horizons Surface Composition Science Theme Team, New Horizons Ralph and LORRI Teams (2019) Impact craters on Pluto and Charon indicate a deficit of small Kuiper belt objects. Science 363:955-959, 1902.10795

Spencer JR, Stern SA, Moore JM, Weaver HA, Singer KN, Olkin CB, Verbiscer AJ, McKinnon WB, Parker JW, Beyer RA, Keane JT, Lauer TR, Porter SB, White OL, Buratti BJ, El-Maarry MR, Lisse CM, Parker AH, Throop HB, Robbins SJ, Umurhan OM, Binzel RP, Britt DT, Buie MW, Cheng AF, Cruikshank DP, Elliott HA, Gladstone GR, Grundy WM, Hill ME, Horanyi M, Jennings DE, Kavelaars JJ, Linscott IR, McComas DJ, McNutt RL, Protopapa S, Reuter DC, Schenk PM, Showalter MR, Young LA, Zangari AM, Abedin AY, Beddingfield CB, Benecchi SD, Bernardoni E, Bierson CJ, Borncamp D, Bray VJ, Chaikin AL, Dhingra RD, Fuentes C, Fuse T, Gay PL, Gwyn SDJ, Hamilton DP, Hofgartner JD, Holman MJ, Howard AD, Howett CJA, Karoji H, Kaufmann DE, Kinczyk M, May BH, Mountain M, Pätzold M, Petit JM, Piquette MR, Reid IN, Reitsema HJ, Runyon KD, Sheppard SS, Stansberry JA, Stryk T, Tanga P, Tholen DJ, Trilling DE, Wasserman LH (2020) The geology and geophysics of Kuiper Belt object (486958) Arrokoth. Science 367(6481):aay3999, DOI 
10.1126/science.aay3999, 2004.00727

Tiscareno MS, Malhotra R (2003) The Dynamics of Known Centaurs. The Astronomical Journal 126(6):3122-3131, DOI 10.1086/379554, astro-ph/0211076

Tiscareno MS, Malhotra R (2009) Chaotic Diffusion of Resonant Kuiper Belt Objects. The Astronomical Journal 138(3):827-837, DOI 10.1088/ 0004-6256/138/3/827,0807.2835

Tsiganis K, Gomes R, Morbidelli A, Levison HF (2005) Origin of the orbital architecture of the giant planets of the Solar System. Nature 435(7041):459 461, DOI 10.1038/nature03539

Volk K, Malhotra R (2008) The Scattered Disk as the Source of the Jupiter Family Comets. The Astrophysical Journal 687(1):714-725, DOI 10.1086/ $591839,0802.3913$

Volk K, Malhotra R (2013) Do Centaurs preserve their source inclinations? Icarus 224(1):66-73, DOI 10.1016/j.icarus.2013.02.016, 1211.2774

Volk K, Murray-Clay R, Gladman B, Lawler S, Bannister MT, Kavelaars JJ, Petit JM, Gwyn S, Alexandersen M, Chen YT, Lykawka PS, Ip W, Lin HW (2016) OSSOS III-Resonant Trans-Neptunian Populations: Constraints from the first quarter of the Outer Solar System Origins Survey. The Astronomical Journal 152(1):23, DOI 10.3847/0004-6256/152/1/23, 1604.08177

Volk KM (2013) Dynamical studies of the Kuiper belt and the Centaurs. PhD thesis, The University of Arizona

Walsh KJ, Morbidelli A, Raymond SN, O'Brien DP, Mandell AM (2011) A low mass for Mars from Jupiter's early gas-driven migration. Nature 475:206209, DOI 10.1038/nature10201, 1201.5177

Wood J, Horner J, Hinse TC, Marsden SC (2017) The Dynamical History of Chariklo and Its Rings. The Astronomical Journal 153(6):245, DOI 10. 3847/1538-3881/aa6981, 1705.02378

Wood J, Horner J, Hinse TC, Marsden SC (2018) Measuring the severity of close encounters between ringed small bodies and planets. Monthly Notices of the Royal Astronomical Society 480(3):4183-4198, DOI 10.1093/mnras/ sty2047

Yu TYM, Murray-Clay R, Volk K (2018) Trans-Neptunian Objects Transiently Stuck in Neptune's Mean-motion Resonances: Numerical Simulations of the Current Population. The Astronomical Journal 156(1):33, DOI 10.3847/ 1538-3881/aac6cd, 1805.08228 